\title{
Zero modes of quantum graph Laplacians and an index theorem
}

\author{
Jens Bolte, Sebastian Egger and Frank Steiner
}

\begin{abstract}
We study zero modes of Laplacians on compact and noncompact metric graphs with general self-adjoint vertex conditions. In the first part of the paper the number of zero modes is expressed in terms of the trace of a unitary matrix $\mathfrak{S}$ that encodes the vertex conditions imposed on functions in the domain of the Laplacian. In the second part a Dirac operator is defined whose square is related to the Laplacian. In order to accommodate Laplacians with negative eigenvalues it is necessary to define the Dirac operator on a suitable Kreĭn space. We demonstrate that an arbitrary, self-adjoint quantum graph Laplacian admits a factorisation into momentum-like operators in a Krel̆n-space setting. As a consequence, we establish an index theorem for the associated Dirac operator and prove that the zero-mode contribution in the trace formula for the Laplacian can be expressed in terms of the index of the Dirac operator.
\end{abstract}

Mathematics Subject Classification (2010). Primary 81Q35; Secondary 58J20, 47B50.

Keywords. Zero modes, index theorem, quantum graph, Krel̆ space.

\section{Introduction}

Index theorems play an important role in differential topology. They relate topological data of a vector bundle over a manifold to spectral data of a differential operator acting on sections in that bundle. They hence reveal the extent to which topological information about a space is encoded in spectra of certain differential operators. In physics such information is very useful when the vector bundles are associated with principal bundles in a gauge theory. This is, e.g., the case in the Aharonov-Casher effect related to the zero modes of a magnetic Schrödinger operator. Central to the formulation of an index theorem is a suitable Dirac operator. Sometimes, however, one is interested in the zero modes of a certain non-negative operator (which could be a Laplacian or a Schrödinger operator) and realises that these are best 
revealed in terms of a Dirac operator squaring to the operator in question. In such a case the question is whether a given operator admits a factorisation in the form $H=A A^{*}$, where $A^{*}$ is the adjoint of an operator $A$.

In a fairly general setting, a Dirac operator is a self-adjoint operator in a Hilbert space $\mathfrak{H}=\mathfrak{H}_{1} \oplus \mathfrak{H}_{2}$ that possesses a representation

$$
D=\left(\begin{array}{cc}
M_{1} & d^{*} \\
d & -M_{2}
\end{array}\right)
$$

with respect to the decomposition of $\mathfrak{H}$ into the sub-Hilbert spaces $\mathfrak{H}_{1 / 2}$. Its (dense) domain $\mathcal{D}:=\mathcal{D}_{d} \oplus \mathcal{D}_{d^{*}}$ splits correspondingly, with $\mathcal{D}_{d} \subseteq \mathfrak{H}_{1}$ and $\mathcal{D}_{d^{*}} \subseteq \mathfrak{H}_{2}$. Furthermore, $d^{*}: \mathcal{D}_{d^{*}} \rightarrow \mathfrak{H}_{1}$ is the adjoint of $d: \mathcal{D}_{d} \rightarrow \mathfrak{H}_{2}$, and the operators $M_{1 / 2}: \mathcal{D}_{M_{1 / 2}} \rightarrow \mathfrak{H}_{1 / 2}$ are self-adjoint, see [1, p. 151] for more details. Assuming that $d M_{1}=M_{2} d$ and $d^{*} M_{2}=M_{1} d^{*}$, the squared Dirac operator is diagonal,

$$
D^{2}=\left(\begin{array}{cc}
d^{*} d+M_{1}^{2} & 0 \\
0 & d d^{*}+M_{2}^{2}
\end{array}\right) .
$$

Often, $d$ will be a differential in a de Rham complex. Then the operators on the diagonal of (1.2) are Laplacians plus, possibly, lower order terms. A simple example would be $\mathfrak{H}_{1}=\mathfrak{H}_{2}$ and $M_{1}=-M_{2}=m \mathbb{1}$. The standard Dirac-Hamiltonian in quantum mechanics (cf. [1, Section 1]) is of this type, where $d$ is the exterior derivative on $\mathbb{R}^{n}$ and $m$ is the mass of the particle. Below we shall treat Dirac operators on metric graphs where, for simplicity, we put $M_{1}=0, M_{2}=0$. The analytical index of the Dirac operator $D$ in (1.1) is defined as (cf. [1, p. 158])

$$
\begin{aligned}
\operatorname{Ind}(D) & :=\operatorname{dim} \operatorname{ker} d-\operatorname{dim} \operatorname{ker} d^{*} \\
& =\operatorname{dim} \operatorname{ker} d^{*} d-\operatorname{dim} \operatorname{ker} d d^{*},
\end{aligned}
$$

where $\operatorname{dim} \operatorname{ker} d^{*}<\infty$ and $\operatorname{dim} \operatorname{ker} d<\infty$ is assumed.

Quantum graphs are models of differential operators on (metric) graphs. They were introduced in [2, 3] as one-dimensional models with a complex structure. It was found that their spectral properties are the same as in classically chaotic quantum systems in that the spectral correlations follow the predictions of random matrix theory. These observations initiated many further numerical and analytical investigations (see, e.g., 44, 5]). Standard Dirac operators on intervals were already considered in [6], and on compact metric graphs in [7, 8]. A trace formula for the spectral density of standard Dirac operators on metric graphs was derived in [8].

The squared Dirac operator (1.2) is obviously non-negative, and the same is true for the operators $d^{*} d$ and $d d^{*}$ on the diagonal. Quantum graph Laplacians, however, may possess finitely many negative eigenvalues. Therefore, not every self-adjoint quantum graph Laplacian can be factorised in the form $d^{*} d$. This can only be achieved if there is no negative spectrum. The factorisation of non-negative Laplacians of certain types was given in [9, 10, 11] and associated index theorems were proven. In [10] self-adjoint realisations 
of quantum graph Laplacians with non-Robin vertex conditions were considered and, using two independent methods, the indices of the associated Dirac operators were calculated. One method utilises the well-known heat-kernel technique, whereas the other method is based on an edge-wise construction of zero modes. The cases covered in [10] do not exhaust all non-negative quantum graph Laplacians. Self-adjoint realisations with Robin vertex conditions, such that the operators are still non-negative, were treated in [11] and an index theorem was proved. In that context Dirac operators

$$
D=\left(\begin{array}{cc}
0 & d^{*} \\
d & 0
\end{array}\right)
$$

respecting the local structure at the vertices, i.e., the topology of the metric graph, were constructed in such a way that the negative Laplacian is given by $-\Delta=d^{*} d$. This required an appropriate de Rham complex involving boundary values of functions at the vertices.

In the case of non-Robin vertex conditions the index of the associated Dirac operator was found to be of the form [10]

$$
\text { Ind }(D)=\frac{1}{2} \operatorname{tr} \mathfrak{S}_{\Delta} \text {. }
$$

Here $\mathfrak{S}_{\Delta}$ is the vertex scattering matrix or, briefly, the $\mathfrak{S}$-matrix, encoding the vertex conditions imposed on functions in the domain of $-\Delta$. An alternative form of the index that was devised in [10] involves the extent to which the vertex conditions are of the Dirichlet-type, and this form of the index theorem was generalised in [11.

Zero modes of quantum graph Laplacians not only play a role in the index (1.3), but also contribute to the trace formula for the Laplacian on a compact graph. In [12] it was shown that

$$
\begin{aligned}
\sum_{k_{n}^{2} \geq 0} h\left(k_{n}\right)= & \mathcal{L} \hat{h}(0)+\gamma h(0)-\frac{1}{4 \pi} \int_{-\infty}^{\infty} h(k) \frac{\operatorname{Im} \operatorname{tr} \mathfrak{S}_{\Delta}(k)}{k} d k \\
& +\sum_{p}\left[\left(\hat{h} * \hat{A}_{p}\right)\left(l_{p}\right)+\left(\hat{h} * \hat{\bar{A}}_{p}\right)\left(l_{p}\right)\right],
\end{aligned}
$$

where $\mathcal{L}$ is the total length of the graph and the sum on the right-hand side extends over all periodic orbits $p$ on the graph; $l_{p}$ is the length of $p$ and $A_{p}(k)$ is an associated amplitude function. The sum on the left-hand side is over all Laplace eigenvalues $k_{n}^{2} \geq 0$, and $h$ is a suitable test function with Fourier transform $\hat{h}$. Crucially, the constant $\gamma \in \mathbb{R}$ in the term summarising the contribution of zero modes could only be determined in terms of the spectral and an algebraic multiplicity of the zero eigenvalue. For Laplacians with non-Robin vertex conditions it was found that

$$
\gamma=\frac{1}{4} \operatorname{tr} \mathfrak{S}_{\Delta}=\frac{1}{2} \operatorname{Ind}(D) .
$$

Hence, for this class of Laplacians an important observation on the role of the graph topology in the trace formula was made. 
When a Laplacian has negative eigenvalues a factorisation $-\Delta=d^{*} d$ as described above is impossible. This has to do with the Hilbert space structure involved, implying that an operator of the form $A A^{*}$ is always non-negative. Therefore, in any attempt at achieving a factorisation of an arbitrary selfadjoint realisation of a quantum graph Laplacian one would have to give up the Hilbert space structure. It is our goal in this paper to provide an approach in which a Dirac operator is defined in a Krel̆ space setting, where the positive-definite inner product is replaced by a non-degenerate hermitian form. This constitutes a minimal deviation from the conventional setting described above such that negative Laplace eigenvalues can still be incorporated. In this context we shall eventually prove an index theorem that is a natural generalisation of the results in [10, 11] and will be presented in the form (1.5).

The paper is organised as follows: We recall the basic definitions and properties of metric graphs and self-adjoint realisations of Laplacians in Section 2. In Section 3] we define spectral and algebraic multiplicities of zero modes and identify a condition under which the two concepts of an algebraic multiplicity coincide. Zero modes of Laplacians are then characterised in Section 4. The trace of the S-matrix is computed in Section 5 and, for compact graphs, used to express the zero-mode contribution in the trace formula (1.6). In Section 6 we present an explicit example of a Dirac operator on an interval in the sense of [11. This will suggest how to generalise the setting in Sections 7 and 8 , There the associated Kreın space structure and Dirac operators are derived and the factorisation of the Laplacians by means of the Dirac operators is constructed. Finally, an index theorem is proved in Section 8 .

\section{Metric graphs and differential Laplacians}

We recall (see, e.g., [13, 14]) that a metric graph $\Gamma=\left(\mathcal{V}, \mathcal{E}_{\text {ex }}, \mathcal{E}_{\text {int }}, \partial, \mathcal{I}\right)$ consists of a finite set of vertices $\mathcal{V}$, a finite set of internal edges $\mathcal{E}_{\text {int }}$, a finite set of external edges $\mathcal{E}_{\text {ex }}$ and a map $\partial$ assigning to every internal edge $e \in \mathcal{E}_{\text {int }}$ an ordered pair of vertices, $\partial(e):=\left(v_{1}, v_{2}\right)$, and to every external edge $e \in \mathcal{E}_{\text {ex }}$ a single vertex, $\partial(e)=v$. The vertices $\partial(e)$ are the edge ends of $e$. Furthermore, to every edge $e \in \mathcal{E}:=\mathcal{E}_{\text {int }} \cup \mathcal{E}_{\text {ex }}$ an interval $I_{e}=\left(0, l_{e}\right)$ is assigned, such that $0<l_{e}<\infty$ if $e \in \mathcal{E}_{\text {int }}$ and $l_{e}=\infty$ if $e \in \mathcal{E}_{\text {ex }}$. The set of these intervals is denoted as $\mathcal{I}$. A metric graph is said to be compact if $\mathcal{E}_{\text {ex }}=\emptyset$, and non-compact otherwise.

We say that an edge $e$ is adjacent to a vertex $v$ if $v \in \partial(e)$, and denote this as $e \sim v$. The degree $d(v)$ of a vertex is the number of edges adjacent to $v$. We emphasise that it is allowed for two vertices to be connected by more than one internal edge, and that the edge ends of an internal edge may coincide, $\partial(e)=(v, v)$, thus producing a loop. A loop counts double in the degree of that vertex. 
A function $\psi$ on $\Gamma$ is a collection of functions on the edges, $\psi=\left\{\psi_{e}\right\}_{e \in \mathcal{E}}$ with $\psi_{e}: I_{e} \rightarrow \mathbb{C}$. Accordingly, function spaces are defined as direct sums, e.g.,

$$
L^{2}(\Gamma):=\bigoplus_{e \in \mathcal{E}} L^{2}\left(0, l_{e}\right) .
$$

Similar constructions apply to Sobolev spaces, $H^{m}(\Gamma)$, and spaces of smooth functions, $C^{\infty}(\Gamma)$, etc. The notation (2.1) in particular implies that no conditions are imposed at the vertices. The space (2.1), equipped with its standard inner product, is a Hilbert space. A function $\psi \in H^{1}(\Gamma)$ has well-defined boundary values at edge ends, which we use to define

$$
\underline{\psi}:=\left(\left\{\psi_{e}(0)\right\}_{e \in \mathcal{E}_{\mathrm{int}}},\left\{\psi_{e}\left(l_{e}\right)\right\}_{e \in \mathcal{E}_{\mathrm{int}}},\left\{\psi_{e}(0)\right\}_{e \in \mathcal{E}_{\mathrm{ex}}}\right)^{T} \in \mathbb{C}^{E}
$$

where $E:=2\left|\mathcal{E}_{\text {int }}\right|+\left|\mathcal{E}_{\text {ex }}\right|$.

Apart from (2.1) we shall also need $p$-form spaces, $p \in\{0,1\}$, as in [11. The 0 -form space for a metric graph $\Gamma$ is $L^{2}(\Gamma)$ and the corresponding 1 -form space is given by $L^{2}(\Gamma) \oplus \mathbb{C}^{E}$. Equipped with their standard inner products the $p$-form spaces are Hilbert spaces.

If $\mathcal{S} \subset \mathbb{C}^{E}$ is a subspace, we denote the corresponding orthogonal projection as $P_{\mathcal{S}}$, and the projection to the orthogonal complement $\mathcal{S}^{\perp}$ of $\mathcal{S}$ as $P_{\mathcal{S}}^{\perp}$. We also define a linear map $I: \mathbb{C}^{E} \rightarrow \mathbb{C}^{E}$ in terms of its matrix representation as

$$
I:=\left(\begin{array}{ccc}
\mathbb{1}_{\mathcal{E}_{\text {int }}} & 0 & 0 \\
0 & -\mathbb{1}_{\mathcal{E}_{\text {int }}} & 0 \\
0 & 0 & \mathbb{1}_{\mathcal{E}_{\text {ex }}}
\end{array}\right) .
$$

We denote the (weak) derivative on $H^{1}(\Gamma)$ as $(\cdot)^{\prime}$, such that $\psi^{\prime}=\left\{\psi_{e}^{\prime}\right\}_{e \in \mathcal{E}}$ with

$$
\psi_{e}^{\prime}=\frac{\mathrm{d} \psi_{e}}{\mathrm{~d} x}
$$

We also sometimes use a momentum operator

$$
p_{\Gamma}:=-\mathrm{i}(\cdot)^{\prime} .
$$

As a differential operator the Laplacian is defined to be

$$
-\Delta \psi:=p_{\Gamma}^{2} \psi=-\psi^{\prime \prime}
$$

for $\psi \in C^{\infty}(\Gamma)$. The self-adjoint realisations of the Laplacian were given in [13, 15, 14]. The parametrisation given in [15] is as follows.

Theorem 2.1. Let $\mathcal{D}_{\Delta} \subset L^{2}(\Gamma)$ be a domain of a self-adjoint realisation of the Laplacian $-\Delta$ with core $C_{0}^{\infty}(\Gamma)$. Then this domain can be uniquely characterised as

$$
\mathcal{D}_{\Delta}=\left\{\psi \in H^{2}(\Gamma) ; \quad(P+L) \underline{\psi}+P^{\perp} I \underline{\psi^{\prime}}=0\right\},
$$

where $P: \mathbb{C}^{E} \rightarrow \mathbb{C}^{E}$ is an orthogonal projection and $L: \mathbb{C}^{E} \rightarrow \mathbb{C}^{E}$ is selfadjoint, satisfying $P^{\perp} L P^{\perp}=L$.

We also denote such a self-adjoint Laplacian $\left(-\Delta, \mathcal{D}_{\Delta}\right)$ as $-\Delta_{P, L}$. 
Definition 2.2. $\quad$ 1. The vertex scattering matrix or, briefly, the $\mathfrak{S}$-matrix, for $-\Delta_{P, L}$ is defined as

$$
\mathfrak{S}_{P, L}(k):=-P-\left(L+\mathrm{i} k P^{\perp}\right)^{-1}\left(L-\mathrm{i} k P^{\perp}\right), \quad k \in \mathbb{C} .
$$

The Laplacian is said to possess $k$-independent vertex conditions if $\mathfrak{S}_{P, L}$ is independent of $k$. Otherwise they are called $k$-dependent.

2. The Laplacian is said to be local with respect to $\Gamma$ if the $\mathfrak{S}$-matrix allows the decomposition

$$
\mathfrak{S}_{P, L}(k)=\oplus_{v \in \mathcal{V}} \mathfrak{S}_{P_{v}, L_{v}}(k), \quad \text { for all } k \in \mathbb{C},
$$

where

$$
\mathfrak{S}_{P_{v}, L_{v}}(k): \mathbb{C}^{d(v)} \rightarrow \mathbb{C}^{d(v)}, \quad \text { for all } \quad v \in \mathcal{V} \quad \text { and } \quad k \in \mathbb{C} .
$$

3. The Laplacian is said to be strictly local with respect to $\Gamma$ if every $\mathfrak{S}_{P_{v}, L_{v}}(k)$ is irreducible, i.e., there exists no refinement of (2.10).

Remark 2.3. - The S-matrix is meromorphic in $\mathbb{C}$ and unitary for $k \in \mathbb{R}$. The pole structure is described in [12].

- For local Laplacians there exist decompositions of the maps $L, P$ and $P^{\perp}$ that correspond to (2.9). Hence, according to the vertex conditions imposed by (2.7), only boundary values of functions on edges that are adjacent to the same vertex are related to each other.

- Non-Robin vertex conditions correspond to the $k$-independent case, where $L=0$, whereas Robin vertex conditions correspond to the $k$ dependent case, with $L \neq 0$.

- As $\operatorname{ran} L \subset \operatorname{ker} P=\operatorname{ran} P^{\perp}$, the projectors $P$ and $P_{\operatorname{ran} L}$ are orthogonal with respect to each other so that $Q:=P+P_{\operatorname{ran} L}$ is also a projector.

Lemma 2.4 ([12]). Let $\mathfrak{S}_{P, L}$ be given and set $Q:=P+P_{\operatorname{ran} L}$. Then

$$
\begin{aligned}
\mathfrak{S}_{\infty} & :=\lim _{k \rightarrow \infty} \mathfrak{S}_{P, L}(k)=P^{\perp}-P, \\
\mathfrak{S}_{0} & :=\lim _{k \rightarrow 0} \mathfrak{S}_{P, L}(k)=Q^{\perp}-Q .
\end{aligned}
$$

In [12] the second line of 2.11) was not explicitly given in this form, but it follows immediately from eq. (3.5) in [12].

\section{Multiplicities of the Laplace-eigenvalue zero}

The principal tool to characterise Laplace eigenvalues is a secular equation [14, 12. In order to set this up one requires the following quantity in addition to the S-matrix.

Definition 3.1. Let $\boldsymbol{l}=\left(l_{1}, \ldots, l_{\left|\mathcal{E}_{\text {int }}\right|}\right)^{T}$ be the vector of finite edge lengths and let $\mathrm{e}^{\mathrm{i} k \boldsymbol{l}}$ be a diagonal matrix with diagonal entries $\mathrm{e}^{\mathrm{i} k l_{e}}, e \in \mathcal{E}_{\text {int. }}$. For $k \in \mathbb{C}$ define the matrix

$$
T(k ; \boldsymbol{l}):=\left(\begin{array}{cc}
T_{\mathrm{int}}(k ; \boldsymbol{l}) & 0 \\
0 & 0_{\mathcal{E}_{\mathrm{ex}}}
\end{array}\right),
$$


acting on $\mathbb{C}^{E}=\mathbb{C}^{2\left|\mathcal{E}_{\text {int }}\right|} \oplus \mathbb{C}^{\left|\mathcal{E}_{\text {ex }}\right|}$, where

$$
T_{\text {int }}(k ; \boldsymbol{l})=\left(\begin{array}{cc}
0 & \mathrm{e}^{\mathrm{i} k \boldsymbol{l}} \\
\mathrm{e}^{\mathrm{i} k \boldsymbol{l}} & 0
\end{array}\right) .
$$

Remark 3.2. When $k \in \mathbb{R}$, the matrix $T_{\text {int }}(k ; \boldsymbol{l})$ is unitary. The same is true for $T(k ; \boldsymbol{l})$ iff $\mathcal{E}_{\mathrm{ex}}=\emptyset$, i.e., for a compact graph.

The secular function is introduced on the basis of the following result [16].

Proposition 3.3. Let $k \in \mathbb{C} \backslash[\mathrm{i} \sigma(L) \cup\{0\}]$, then $k^{2}$ is an eigenvalue of $-\Delta_{P, L}$ with spectral multiplicity $g$, iff 1 is an eigenvalue of the matrix $\mathfrak{S}_{P, L}(k) T(k ; \boldsymbol{l})$ with geometric multiplicity $g$.

Hence, the secular function

$$
F(k):=\operatorname{det}\left(\mathbb{1}_{\mathbb{C}^{E}}-\mathfrak{S}_{P, L}(k) T(k ; \boldsymbol{l})\right), \quad k \in \mathbb{C} \backslash[\mathrm{i} \sigma(L) \cup\{0\}],
$$

has a zero at $k$, iff $k^{2}$ is an eigenvalue of $-\Delta_{P, L}$. Setting $l_{\min }:=\min \left\{l_{e} ; \quad e \in\right.$ $\left.\mathcal{E}_{\text {int }}\right\}$ and

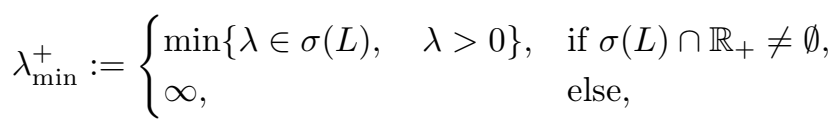

it was shown in [12] that on a compact graph $\Gamma$ the spectral multiplicity of a Laplace eigenvalue $k^{2} \neq 0$ coincides with the order of the zero $k$ of the secular function $F$, if one assumes that $l_{\min }>2 / \lambda_{\min }^{+}$.

In the following we shall extend the analysis to $k=0$ and to noncompact $\Gamma$. We shall also clarify the role of the additional assumption. For this we require the following notions.

Definition 3.4. $\quad$ - A zero mode of the Laplacian $-\Delta_{P, L}$ is a non-trivial element of ker $\Delta_{P, L} \subset \mathcal{D}_{\Delta}$. The spectral multiplicity of the eigenvalue zero is

$$
g_{0}:=\operatorname{dim} \operatorname{ker} \Delta_{P, L} .
$$

- The algebraic multiplicity $N$ of the eigenvalue zero in the sense of [10] is the order of the zero of $F$ at $k=0$,

$$
N:=\left.\operatorname{ord}[F]\right|_{k=0} \text {. }
$$

- The algebraic multiplicity $\tilde{N}$ of the eigenvalue zero in the sense of 17 is

$$
\widetilde{N}:=\operatorname{dim} \operatorname{ker}\left(\mathbb{1}_{\mathbb{C}^{E}}-\mathfrak{S}_{0} \mathfrak{J}_{\mathcal{E}}\right),
$$

where

$$
\mathfrak{J}_{\mathcal{E}}:=\left(\begin{array}{cc}
J_{\mathcal{E}_{\text {int }}} & 0 \\
0 & 0 \\
\mathcal{E}_{\text {ex }}
\end{array}\right), \quad J_{\mathcal{E}_{\text {int }}}:=\left(\begin{array}{cc}
0 & \mathbb{1}_{\mathcal{E}_{\text {int }}} \\
\mathbb{1}_{\mathcal{E}_{\text {int }}} & 0
\end{array}\right) .
$$

As $T(0 ; \boldsymbol{l})=\mathfrak{J}_{\mathcal{E}}$, comparing $F(0)$ with (3.7) one may be tempted to assume that $N=\widetilde{N}$. The following example, however, shows that this need not be the case. 
Example. Consider a graph consisting of one edge and two vertices, to which the interval $I=[0, l]$ is assigned. Choose $P=0$ and $L=\lambda \mathbb{1}_{\mathbb{C}^{2}}, \lambda \in \mathbb{R} \backslash\{0\}$, implying Robin conditions at the vertices. Using that any zero mode is of the form $\psi_{0}(x)=\alpha x+\beta$ and imposing the vertex conditions gives the spectral multiplicity of the eigenvalue zero as

$$
g_{0}= \begin{cases}1, & \text { if } l=\frac{2}{\lambda}, \\ 0, & \text { else. }\end{cases}
$$

Furthermore, $\mathfrak{S}_{0}=-\mathbb{1}_{\mathbb{C}^{2}}$ leads to the secular function

$$
F(k)=1-\left(\frac{\lambda-\mathrm{i} k}{\lambda+\mathrm{i} k}\right)^{2} \mathrm{e}^{2 \mathrm{i} k l} .
$$

Hence,

$$
N=\left.\operatorname{ord}[F]\right|_{k=0}= \begin{cases}3, & \text { if } l=\frac{2}{\lambda} \\ 1, & \text { else }\end{cases}
$$

However,

$$
\widetilde{N}=\operatorname{dim} \operatorname{ker}\left(\begin{array}{ll}
1 & 1 \\
1 & 1
\end{array}\right)=1
$$

Under the additional assumption $l>2 / \lambda$ required in 12 (see also the paragraph below (3.4)), one finds that $N=\tilde{N}$. This is not true when $l=2 / \lambda$. However, the quantity $\gamma$ in (1.6), which was determined in 12 to be $\gamma=g_{0}-N / 2$, satisfies

$$
\gamma=\frac{1}{4} \operatorname{tr} \mathfrak{S}_{0}=-\frac{1}{2}
$$

in all cases of this example.

In the following we shall prove that the first equality in 3.13) holds for all self-adjoint Laplacians on compact graphs. Before, we shall derive a sufficient criterion for $N=\widetilde{N}$ to hold, which in the example above simply excludes the case $l=2 / \lambda$. For this we require certain subspaces of $\mathbb{C}^{E}$.

Definition 3.5. Define the following subspaces of the space $\mathbb{C}^{E}$ of boundary values:

$$
\begin{aligned}
& M_{\mathrm{sy}}:=\left\{\left(\boldsymbol{c}, \boldsymbol{c}, \mathbf{0}_{\mathcal{E}_{\mathrm{ex}}}\right)^{T} \in \mathbb{C}^{E}, \quad \boldsymbol{c} \in \mathbb{C}^{\left|\mathcal{E}_{\mathrm{int}}\right|}\right\}, \\
& M_{\text {asy }}:=\left\{\left(\boldsymbol{c},-\boldsymbol{c}, \mathbf{0}_{\mathcal{E}_{\text {ex }}}\right)^{T} \in \mathbb{C}^{E}, \quad \boldsymbol{c} \in \mathbb{C}^{\left|\mathcal{E}_{\text {int }}\right|}\right\}, \\
& M_{0}:=\left\{\left(\mathbf{0}_{\mathcal{E}_{\mathrm{int}}}, \mathbf{0}_{\mathcal{E}_{\mathrm{int}}}, \boldsymbol{c}\right)^{T} \in \mathbb{C}^{E}, \quad \boldsymbol{c} \in \mathbb{C}^{\left|\mathcal{E}_{\mathrm{ex}}\right|}\right\} .
\end{aligned}
$$

We also set

$$
M:=M_{\mathrm{sy}} \oplus M_{\mathrm{asy}} .
$$


The projectors onto $M_{\mathrm{sy}}, M_{\text {asy }}$ and $M_{0}$ are denoted as $P_{\mathrm{sy}}, P_{\text {asy }}$ and $P_{0}$, respectively. In matrix form the first two read

$$
P_{\mathrm{sy}}=\frac{1}{2}\left(\begin{array}{ccc}
\mathbb{1}_{\mathcal{E}_{\text {int }}} & \mathbb{1} & 0 \\
\mathbb{1} & \mathbb{1}_{\mathcal{E}_{\text {int }}} & 0 \\
0 & 0 & 0_{\mathcal{E}_{\text {ex }}}
\end{array}\right), \quad P_{\text {asy }}=\frac{1}{2}\left(\begin{array}{ccc}
\mathbb{1}_{\mathcal{E}_{\text {int }}} & -\mathbb{1} & 0 \\
-\mathbb{1} & \mathbb{1}_{\mathcal{E}_{\text {int }}} & 0 \\
0 & 0 & 0 \\
\mathcal{E}_{\text {ex }}
\end{array}\right),
$$

such that

$$
P_{\text {sy }}-P_{\text {asy }}=\mathfrak{J}_{\mathcal{E}}=T(0 ; \boldsymbol{l}),
$$

see $(3.8)$.

We need to consider the eigenvalue problem of the matrix

$$
U(k):=\mathfrak{S}_{P, L}(k) T(k ; \boldsymbol{l}), \quad k \in \mathbb{R} .
$$

Lemma 3.6. Let $k \in \mathbb{R}$. In the eigenvalue problem

$$
U(k) \boldsymbol{w}=\lambda \boldsymbol{w},
$$

where, in general, $\boldsymbol{w} \in \mathbb{C}^{E}$ and $\lambda \in \mathbb{C}$, the following equivalence holds:

$$
\boldsymbol{w} \in M \Leftrightarrow|\lambda|=1 \text {. }
$$

Furthermore, $M_{0}$ is the eigenspace corresponding to the eigenvalue $\lambda=0$.

Proof. From the unitarity of $\mathfrak{S}_{P, L}(k)$ for $k \in \mathbb{R}$ and from (3.1) one infers that

$$
\left\|\mathfrak{S}_{P, L}(k) T(k ; \boldsymbol{l}) \boldsymbol{w}\right\|_{\mathbb{C}^{E}} \leq\|\boldsymbol{w}\|_{\mathbb{C}^{E}},
$$

implying $|\lambda| \leq 1$. Moreover, a strict inequality holds iff $P_{0} \boldsymbol{w} \neq \mathbf{0}$.

It is obvious from (3.1) that $T(k ; \boldsymbol{l}) \boldsymbol{w}=\mathbf{0}$ iff $\boldsymbol{w} \in M_{0}$, implying the last claim.

In general, when $\Gamma$ is non-compact and hence $\mathcal{E}_{\text {ex }} \neq \emptyset$, the matrix $U(k)=$ $\mathfrak{S}_{P, L}(k) T(k ; \boldsymbol{l})$ is neither unitary nor can it be diagonalised. Its eigenvectors do not span the entire space $\mathbb{C}^{E}$ and eigenvectors corresponding to different eigenvalues are not orthogonal with respect to each other. The following observation, however, will turn out to be sufficient.

Lemma 3.7. Let $k \in \mathbb{R}$ and $\lambda(k)$ be an eigenvalue of $U(k)$. If $|\lambda(k)|=1$ the eigenvalue is semi-simple.

Proof. Due to Lemma 3.6 any eigenvector $\boldsymbol{w}$ corresponding to $\lambda(k)$ with $|\lambda(k)|=1$ is in $M$. We now modify $U(k)$ on the orthogonal complement $M^{\perp}=M_{0}$ to this subspace by setting

$$
\widetilde{U}(k):=\mathfrak{S}_{P, L}(k) \widetilde{T}(k ; \boldsymbol{l}),
$$

where

$$
\widetilde{T}(k ; \boldsymbol{l}):=\left(\begin{array}{cc}
T_{\mathrm{int}}(k ; \boldsymbol{l}) & 0 \\
0 & \mathbb{1}_{\mathcal{E}_{\mathrm{ex}}}
\end{array}\right) .
$$

When $k \in \mathbb{R}$ the matrix $\widetilde{U}(k)$ is unitary. Therefore, its eigenvalues $\widetilde{\lambda}(k)$ satisfy $|\widetilde{\lambda}(k)|=1$, and there exists an orthonormal basis of eigenvectors $\left\{\boldsymbol{x}_{1}, \ldots, \boldsymbol{x}_{E}\right\}$ 
for $\mathbb{C}^{E}$. We arrange this basis in such a way that $\boldsymbol{x}_{1}, \ldots, \boldsymbol{x}_{F} \in M$ (where $\left.1 \leq F \leq 2\left|\mathcal{E}_{\text {int }}\right|\right)$, and set

$$
\widetilde{M}:=\operatorname{lin}\left\{\boldsymbol{x}_{l} ; \quad 1 \leq l \leq F\right\} \subset M .
$$

As $U(k)$ and $\widetilde{U}(k)$ coincide on $M$, the eigenvectors $\boldsymbol{x}_{l} \in \widetilde{M}$ are also eigenvectors of $U(k)$ with the same eigenvalues. In fact, by Lemma 3.6 they exhaust all eigenvectors of $U(k)$ with eigenvalues satisfying $|\lambda(k)|=1$.

By construction, $\widetilde{U}(k)$ leaves $\widetilde{M}$ and $\widetilde{M} \perp$ invariant,

$$
\widetilde{U}(k)=P_{\widetilde{M}} \widetilde{U}(k) P_{\widetilde{M}}+P_{\widetilde{M}}^{\perp} \widetilde{U}(k) P_{\widetilde{M}}^{\perp},
$$

where $P_{\widetilde{M}}$ and $P \underset{\widetilde{M}}{\perp}$ are the projectors onto $\widetilde{M}$ and $\widetilde{M}^{\perp}$, respectively. This then implies that

$$
U(k) \boldsymbol{x}=\widetilde{U}(k) \boldsymbol{x} \in \widetilde{M}, \quad \text { for every } \quad \boldsymbol{x} \in \widetilde{M} \subset M,
$$

so that $U(k)$ leaves $\widetilde{M}$ invariant, too. Now let $\boldsymbol{x} \in \widetilde{M^{\perp}}$. Since $P_{0} \boldsymbol{x} \in M_{0}=$ $M^{\perp} \subset \widetilde{M^{\perp}}$ and $T P_{0} \boldsymbol{x}=\mathbf{0}$, one obtains

$$
U(k) \boldsymbol{x}=U(k)\left(\boldsymbol{x}-P_{0} \boldsymbol{x}\right)=\widetilde{U}(k)\left(\boldsymbol{x}-P_{0} \boldsymbol{x}\right) \in \widetilde{M}^{\perp},
$$

so that $U(k)$ also leaves $\widetilde{M}^{\perp}$ invariant. Hence,

$$
U(k)=P_{\widetilde{M}} U(k) P_{\widetilde{M}}+P_{\widetilde{M}}^{\perp} U(k) P_{\widetilde{M}}^{\perp} .
$$

We therefore conclude that the Jordan blocks in the Jordan normal forms of $P_{\widetilde{M}}(k) \widetilde{U}(k) P_{\widetilde{M}}(k)$ and $P_{\widetilde{M}}(k) U(k) P_{\widetilde{M}}(k)$ coincide. As the eigenvectors of $U(k)$ corresponding to eigenvalues with $|\lambda(k)|=1$ are in $\widetilde{M}$, and $\widetilde{U}(k)$ can be diagonalised, these eigenvalues are semi-simple.

Together with Theorem 1 in [18, p. 402], Lemma 3.7 ensures that any eigenvalue (-function) $\lambda(k)$ of $U(k)$ such that there exists $k_{0}$ with $\left|\lambda\left(k_{0}\right)\right|=1$ is (real) differentiable in a neighbourhood of $k_{0}$. Furthermore,

$$
\lambda(k)=\lambda\left(k_{0}\right)+\sum_{n=l}^{\infty} a_{n}\left(k-k_{0}\right)^{n / l},
$$

where $a_{n} \in \mathbb{C}$ and $l$ is an integer not exceeding the multiplicity of the eigenvalue $\lambda\left(k_{0}\right)$. There also exists a corresponding eigenvalue (-function) $\boldsymbol{x}(k)$ with an expansion

$$
\boldsymbol{x}(k)=\sum_{n=0}^{\infty} \boldsymbol{x}_{n}\left(k-k_{0}\right)^{n / l},
$$

that converges for $0<\left|k-k_{0}\right|<\delta$ with some $\delta>0$. Moreover, $\boldsymbol{x}_{n} \in$ $\operatorname{ker}\left(U\left(k_{0}\right)-\lambda\left(k_{0}\right)\right)$ for $n=0, \ldots, l-1$.

The above results enable us to prove an equivalent to Lemma 4.5 in [12], although in the present case $U(k)$ is not unitary. For this we require the 
following matrices,

$$
\mathfrak{D}(\boldsymbol{l}):=\left(\begin{array}{ccc}
D(\boldsymbol{l}) & 0 & 0 \\
0 & D(\boldsymbol{l}) & 0 \\
0 & 0 & 0 \\
\mathcal{E}_{\mathrm{ex}}
\end{array}\right) \text {, with } \quad D(\boldsymbol{l}):=\left(\begin{array}{ccc}
l_{1} & & 0 \\
& \ddots & \\
0 & & l_{\left|\mathcal{E}_{\mathrm{int}}\right|}
\end{array}\right) .
$$

Lemma 3.8. Let $\lambda(k)$ be an eigenvalue function of $U(k)$ and assume that there exists $k_{0} \in \mathbb{R}$ such that $\lambda\left(k_{0}\right)=1$. Then $\lambda(k)$ is real differentiable at $k_{0}$ and there exists a vector $\boldsymbol{x}_{0} \in \operatorname{ker}\left(\mathbb{1}_{\mathcal{E}}-U\left(k_{0}\right)\right)$ such that

$$
\mathrm{i} \lambda^{\prime}\left(k_{0}\right)=2\left\langle\boldsymbol{x}_{0}, \frac{L}{L^{2}+k_{0}^{2}} \boldsymbol{x}_{0}\right\rangle_{\mathbb{C}^{E}}-\left\langle\boldsymbol{x}_{0}, \mathfrak{D}(\boldsymbol{l}) \boldsymbol{x}_{0}\right\rangle_{\mathbb{C}^{E}} .
$$

Proof. A calculation that can be copied verbatim from the proof of Lemma 4.5 in 12 yields

$$
\begin{aligned}
& \mathrm{i}\left\langle\boldsymbol{x}(k),\left(\frac{-2 L}{L^{2}+k^{2}} U(k)+U(k) \mathfrak{D}(\boldsymbol{l})\right) \boldsymbol{x}(k)\right\rangle_{\mathbb{C}^{E}}+\left\langle\boldsymbol{x}(k), U(k) \boldsymbol{x}^{\prime}(k)\right\rangle_{\mathbb{C}^{E}} \\
& =\lambda^{\prime}(k)\langle\boldsymbol{x}(k), \boldsymbol{x}(k)\rangle_{\mathbb{C}^{E}}+\lambda(k)\left\langle\boldsymbol{x}(k), \boldsymbol{x}^{\prime}(k)\right\rangle_{\mathbb{C}^{E}} .
\end{aligned}
$$

Since $U(k)$ is real analytic,

$$
U(k)=U\left(k_{0}\right)+O\left(k-k_{0}\right), \quad U(k)^{*}=U\left(k_{0}\right)^{*}+O\left(k-k_{0}\right),
$$

holds for $k \rightarrow k_{0}$.

From (3.31) one obtains that $\lambda(k)=1+O\left(k-k_{0}\right)$, and from (3.32) that

$$
\boldsymbol{x}^{\prime}(k)=\sum_{n=1}^{l} \frac{n}{l} \boldsymbol{x}_{n}\left(k-k_{0}\right)^{(n-l) / l}+O\left(\left(k-k_{0}\right)^{1 / l}\right),
$$

where $\boldsymbol{x}_{n} \in \operatorname{ker}\left(U\left(k_{0}\right)-\lambda\left(k_{0}\right) \mathbb{1}\right)$ for $n=0, \ldots, l-1$. We hence find that

$$
\begin{aligned}
&\left\langle\boldsymbol{x}(k), U(k) \boldsymbol{x}^{\prime}(k)\right\rangle_{\mathbb{C}^{E}} \\
&=\sum_{m=0}^{l-1} \sum_{n=1}^{l} \frac{n}{l}\left(k-k_{0}\right)^{(m+n-l) / l}\left\langle\boldsymbol{x}_{m}, \boldsymbol{x}_{n}\right\rangle_{\mathbb{C}^{E}}+O\left(\left(k-k_{0}\right)^{1 / l}\right),
\end{aligned}
$$

as well as

$$
\begin{aligned}
& \lambda(k)\left\langle\boldsymbol{x}(k), \boldsymbol{x}^{\prime}(k)\right\rangle_{\mathbb{C}^{E}} \\
&=\sum_{m=0}^{l-1} \sum_{n=1}^{l-1} \frac{n}{l}\left(k-k_{0}\right)^{(m+n-l) / l}\left\langle\boldsymbol{x}_{m}, \boldsymbol{x}_{n}\right\rangle_{\mathbb{C}^{E}}+O\left(\left(k-k_{0}\right)^{1 / l}\right) .
\end{aligned}
$$

Notice that the first terms on the right-hand side of (3.38) and (3.39) coincide, whereas the error terms, in general, are only of the same order. A similar calculation reveals that

$$
\langle\boldsymbol{x}(k), U(k) \mathfrak{D}(\boldsymbol{l}) \boldsymbol{x}(k)\rangle_{\mathbb{C}^{E}}=\left\langle\boldsymbol{x}_{0}, \mathfrak{D}(\boldsymbol{l}) \boldsymbol{x}_{0}\right\rangle_{\mathbb{C}^{E}}+O\left(\left(k-k_{0}\right)^{1 / l}\right) .
$$


Subtracting the first terms on the right-hand side of (3.38) and (3.39), respectively, from (3.35), one can perform the limit $k \rightarrow k_{0}$ and thus obtains the identity (3.34).

We now address the question of relating the three multiplicities of the (Laplace-) eigenvalue zero introduced in Definition 3.4. Regarding the quantity $N$ from $(3.6)$ we need to ensure that $\lambda^{\prime}(0) \neq 0$ for all eigenvalue functions $\lambda(k)$ with $\lambda(0)=1$. On the other hand, a characterisation of $\widetilde{N}$ from (3.7) requires the knowledge of $\operatorname{ker}(\mathbb{1}-U(0))$. For the latter question we notice that $U(0)=\mathfrak{S}_{0} T(0 ; \boldsymbol{l})=\left(Q^{\perp}-Q\right) \mathfrak{J}_{\mathcal{E}}$, see (2.11), (3.1) and (3.8).

Guided by the fact that $\mathfrak{S}_{0}=Q^{\perp}-Q$, we now assume that $Q$ is any orthogonal projector in $\mathbb{C}^{E}$ and define

$$
\mathfrak{S}_{Q}:=Q^{\perp}-Q .
$$

For this quantity we find the following result.

Lemma 3.9. Let $\mathfrak{S}_{Q}$ be as in (3.41), then

$$
\begin{aligned}
& \mathfrak{S}_{Q} \mathfrak{J}_{\mathcal{E}} \boldsymbol{w}=-\boldsymbol{w} \quad \Leftrightarrow \quad \boldsymbol{w} \in\left(\operatorname{ker} Q \cap M_{\text {asy }}\right) \oplus\left((\operatorname{ker} Q)^{\perp} \cap M_{\mathrm{sy}}\right), \\
& \mathfrak{S}_{Q} \mathfrak{J}_{\mathcal{E}} \boldsymbol{w}=\boldsymbol{w} \quad \Leftrightarrow \quad \boldsymbol{w} \in\left((\operatorname{ker} Q)^{\perp} \cap M_{\text {asy }}\right) \oplus\left(\operatorname{ker} Q \cap M_{\mathrm{sy}}\right) .
\end{aligned}
$$

Proof. It follows from Lemma 3.6 that in both cases $\boldsymbol{w} \in M$. An arbitrary vector $\boldsymbol{w} \in M$ can be uniquely decomposed as

$$
\boldsymbol{w}=\boldsymbol{w}_{\mathrm{sy}}+\boldsymbol{w}_{\mathrm{asy}}, \quad \boldsymbol{w}_{\mathrm{sy}} \in M_{\mathrm{sy}}, \quad \boldsymbol{w}_{\mathrm{asy}} \in M_{\mathrm{asy}} .
$$

Decomposing further,

$$
\begin{gathered}
\boldsymbol{w}_{\mathrm{sy}}=\boldsymbol{w}_{\mathrm{sy}}^{1}+\boldsymbol{w}_{\mathrm{sy}}^{2}, \quad \boldsymbol{w}_{\mathrm{sy}}^{1} \in \operatorname{ker} Q, \quad \boldsymbol{w}_{\mathrm{sy}}^{2} \in(\operatorname{ker} Q)^{\perp}, \\
\boldsymbol{w}_{\mathrm{asy}}=\boldsymbol{w}_{\text {asy }}^{1}+\boldsymbol{w}_{\mathrm{asy}}^{2}, \quad \boldsymbol{w}_{\mathrm{asy}}^{1} \in \operatorname{ker} Q, \quad \boldsymbol{w}_{\text {asy }}^{2} \in(\operatorname{ker} Q)^{\perp},
\end{gathered}
$$

and noting that due to (3.19),

$$
\mathfrak{S}_{Q} \mathfrak{J}_{\mathcal{E}}=Q^{\perp} P_{\mathrm{sy}}+Q P_{\text {asy }}-Q^{\perp} P_{\text {asy }}-Q P_{\text {sy }}
$$

we obtain that $\mathfrak{S}_{Q} \mathfrak{J}_{\mathcal{E}} \boldsymbol{w}=\boldsymbol{w}$ reads

$$
\begin{gathered}
\left(Q^{\perp} P_{\mathrm{sy}}+Q P_{\mathrm{asy}}-Q^{\perp} P_{\mathrm{asy}}-Q P_{\mathrm{sy}}\right)\left(\boldsymbol{w}_{\mathrm{sy}}+\boldsymbol{w}_{\mathrm{asy}}\right) \\
=\boldsymbol{w}_{s y}^{1}+\boldsymbol{w}_{s y}^{2}+\boldsymbol{w}_{a s y}^{1}+\boldsymbol{w}_{a s y}^{2},
\end{gathered}
$$

or

$$
\boldsymbol{w}_{\mathrm{sy}}^{1}-\boldsymbol{w}_{\mathrm{sy}}^{2}-\boldsymbol{w}_{\text {asy }}^{1}+\boldsymbol{w}_{\text {asy }}^{2}=\boldsymbol{w}_{\text {sy }}^{1}+\boldsymbol{w}_{\text {sy }}^{2}+\boldsymbol{w}_{\text {asy }}^{1}+\boldsymbol{w}_{\text {asy }}^{2} .
$$

Hence, $\boldsymbol{w}_{\text {sy }}^{2}+\boldsymbol{w}_{\text {asy }}^{1}=\mathbf{0}$. Due to the linear independence of $\boldsymbol{w}_{\text {sy }}^{2}$ and $\boldsymbol{w}_{\text {asy }}^{1}$ and with a similar reasoning for the eigenvalue -1 the claims in the Lemma follow.

Corollary 3.10. It follows immediately that

$$
\operatorname{ker}(\mathbb{1}-U(0))=\left((\operatorname{ker} Q)^{\perp} \cap M_{\text {asy }}\right) \oplus\left(\operatorname{ker} Q \cap M_{\text {sy }}\right),
$$

and hence that

$$
\widetilde{N}=\operatorname{dim}\left((\operatorname{ker} Q)^{\perp} \cap M_{\text {asy }}\right)+\operatorname{dim}\left(\operatorname{ker} Q \cap M_{\text {sy }}\right) .
$$


For a characterisation of $N$ we need to consider the derivative (3.34) at $k_{0}=0$, since $N=\widetilde{N}$ iff $\lambda^{\prime}(0) \neq 0$ for every eigenvalue of $U$ with $\lambda(0)=1$. The first term on the right-hand side of (3.34) has to be treated with some care when $k_{0}=0$ as $L$ is, in general, not invertible. However, any normal map $A: \mathbb{C}^{n} \rightarrow \mathbb{C}^{n}$ has a Moore-Bjerhammer-Penrose pseudo-inverse that is defined as follows: Denote by $E_{0}$ the zero-eigenspace and introduce the orthogonal decomposition $\mathbb{C}^{n}=E_{0} \oplus E_{0}^{\perp}$. Then $A_{\mathrm{MBP}}^{-1}:=0_{E_{0}} \oplus\left(\left.A\right|_{E_{0}^{\perp}}\right)^{-1}$, i.e., $A$ is inverted on the non-zero eigenspaces only. We also require the following matrix,

$$
G(\boldsymbol{l}):=\left(\begin{array}{ccc}
D(\boldsymbol{l})^{-1} & -D(\boldsymbol{l})^{-1} & 0 \\
-D(\boldsymbol{l})^{-1} & D(\boldsymbol{l})^{-1} & 0 \\
0 & 0 & 0 \\
\mathcal{E}_{\mathrm{ex}}
\end{array}\right)
$$

where $D(\boldsymbol{l})$ is defined in 3.33 . With $(\underline{3.18}$ ) and 3.33 one obtains that

$$
G(\boldsymbol{l})=2 P_{\text {asy }} \mathfrak{D}(\boldsymbol{l})_{\mathrm{MBP}}^{-1}=2 P_{\text {asy }} \mathfrak{D}(\boldsymbol{l})_{\mathrm{MBP}}^{-1} P_{\text {asy }} .
$$

Lemma 3.11. The eigenvalues of $L_{\mathrm{MBP}}^{-1} G(\boldsymbol{l})$ are real. Moreover, if $l_{\mathrm{min}}>$ $2 / \lambda_{\min }^{+}$, the largest eigenvalue $\tau_{\max }$ of $L_{\mathrm{MBP}}^{-1} G(\boldsymbol{l})$ satisfies $\tau_{\max }<1$.

Proof. We define

$$
\mathfrak{M}:=2 \mathfrak{D}(\boldsymbol{l})_{\mathrm{MBP}}^{-1 / 2} P_{\text {asy }} L_{\mathrm{MBP}}^{-1} P_{\text {asy }} \mathfrak{D}(\boldsymbol{l})_{\mathrm{MBP}}^{-1 / 2},
$$

and notice that $\mathfrak{M}$ is self-adjoint. Through a cyclic permutation of the factors, $\mathfrak{M}$ can be transformed into $L_{\mathrm{MBP}}^{-1} G(\boldsymbol{l})$, see (3.51). Hence the non-zero spectrum of $\mathfrak{M}$ coincides with that of $L_{\mathrm{MBP}}^{-1} G(\boldsymbol{l})$. Thus the eigenvalues of $L_{\mathrm{MBP}}^{-1} G(\boldsymbol{l})$ are real.

Let $\tau_{\max }$ be the largest eigenvalue of $\mathfrak{M}$ with associated normalised eigenvector $\boldsymbol{x}$, then

$$
\begin{aligned}
\tau_{\max } & =\left\langle\boldsymbol{x}, 2 \mathfrak{D}(\boldsymbol{l})_{\mathrm{MBP}}^{-1 / 2} P_{\mathrm{asy}} L_{\mathrm{MBP}}^{-1} P_{\mathrm{asy}} \mathfrak{D}(\boldsymbol{l})_{\mathrm{MBP}}^{-1 / 2} \boldsymbol{x}\right\rangle_{\mathbb{C}^{E}} \\
& =2\left\langle P_{\mathrm{asy}} \mathfrak{D}(\boldsymbol{l})_{\mathrm{MBP}}^{-1 / 2} \boldsymbol{x}, L_{\mathrm{MBP}}^{-1} P_{\mathrm{asy}} \mathfrak{D}(\boldsymbol{l})_{\mathrm{MBP}}^{-1 / 2} \boldsymbol{x}\right\rangle_{\mathbb{C}^{E}} \\
& \leq 2\left\|\mathfrak{D}(\boldsymbol{l})_{\mathrm{MBP}}^{-1 / 2} \boldsymbol{x}\right\|_{\mathbb{C}^{E}}^{2} \frac{1}{\lambda_{\min }^{+}} \\
& \leq \frac{2}{l_{\mathrm{min}} \lambda_{\min }^{+}} .
\end{aligned}
$$

Hence, the assumption $l_{\min }>2 / \lambda_{\min }^{+}$implies $\tau_{\max }<1$.

We remark that the condition $l_{\min }>2 / \lambda_{\min }^{+}$is sufficient for $\tau_{\max }<1$, but not necessary. As an example, choose $L$ such that $L_{\mathrm{MBP}}^{-1}=P_{\mathrm{sy}}$. Then $L_{\mathrm{MBP}}^{-1} G(\boldsymbol{l})=0$, independent of the choice of edge lengths.

Proposition 3.12. Assume that $\tau_{\max }<1$, where $\tau_{\max }$ is the largest eigenvalue of $L_{\mathrm{MBP}}^{-1} G(\boldsymbol{l})$. Then $N=\tilde{N}$. 
Proof. When $k_{0}=0$, the relation 3.34 reads

$$
\mathrm{i} \lambda^{\prime}(0)=2\left\langle\boldsymbol{x}_{0}, L_{\mathrm{MBP}}^{-1} \boldsymbol{x}_{0}\right\rangle_{\mathbb{C}^{E}}-\left\langle\boldsymbol{x}_{0}, \mathfrak{D}(\boldsymbol{l}) \boldsymbol{x}_{0}\right\rangle_{\mathbb{C}^{E}} .
$$

Since $\boldsymbol{x}_{0} \in \operatorname{ker}(\mathbb{1}-U(0))$, the relation (3.42) together with $\operatorname{ran} L \subset \operatorname{ker} Q^{\perp}$ implies that $L_{\mathrm{MBP}}^{-1} \boldsymbol{x}_{0}=P_{\mathrm{asy}} L_{\mathrm{MBP}}^{-1} P_{\text {asy }} \boldsymbol{x}_{0}$. We define $\boldsymbol{y}_{0}:=\mathfrak{D}(\boldsymbol{l})^{1 / 2} \boldsymbol{x}_{0}$ and notice that, as $\boldsymbol{x}_{0} \in M$, this can be inverted to yield $\boldsymbol{x}_{0}=\mathfrak{D}(\boldsymbol{l})_{\mathrm{MBP}}^{-1 / 2} \boldsymbol{y}_{0}$. Altogether, this implies

$$
\begin{aligned}
\mathrm{i} \lambda^{\prime}(0) & =\left\langle\boldsymbol{y}_{0}, 2 \mathfrak{D}(\boldsymbol{l})_{\mathrm{MBP}}^{-1 / 2} P_{\mathrm{asy}} L_{\mathrm{MBP}}^{-1} P_{\mathrm{asy}} \mathfrak{D}(\boldsymbol{l})_{\mathrm{MBP}}^{-1 / 2} \boldsymbol{y}_{0}\right\rangle_{\mathbb{C}^{E}}-\left\langle\boldsymbol{y}_{0}, \boldsymbol{y}_{0}\right\rangle_{\mathbb{C}^{E}} \\
& =\left\langle\boldsymbol{y}_{0}, \mathfrak{M} \boldsymbol{y}_{0}\right\rangle-\left\langle\boldsymbol{y}_{0}, \boldsymbol{y}_{0}\right\rangle_{\mathbb{C}^{E}}
\end{aligned}
$$

Since by the proof of Lemma 3.11 the largest eigenvalue is $\tau_{\max }$, we conclude that $\lambda^{\prime}(0) \neq 0$.

The secular function can be written as

$$
F(k)=\tilde{F}(k) \prod_{j=1}^{\tilde{N}}\left(1-\lambda_{j}(k)\right),
$$

where the $\lambda_{j}(k)$ are the eigenvalue functions of $U(k)$ with $\lambda_{j}(0)=1$, and $\tilde{F}(0) \neq 0$. Expanding $\lambda_{j}(k)$ for small $|k|$ then shows that $\lambda_{j}^{\prime}(0) \neq 0$ implies $N=\left.\operatorname{ord}[F]\right|_{k=0}=\tilde{N}$.

Under the assumption in the Proposition one can therefore speak of the algebraic multiplicity of the Laplace eigenvalue zero.

\section{Zero modes}

In [17] zero modes were characterised in terms of the quadratic form associated with the Laplacian $-\Delta_{P, L}$,

$$
\left\|\psi^{\prime}\right\|_{L^{2}(\Gamma)}^{2}-\langle\underline{\psi}, L \underline{\psi}\rangle_{\mathbb{C}^{E}}=0 .
$$

For non-Robin vertex conditions, when $L=0$, it follows that a zero mode must be constant on each edge, i.e.,

$$
\psi_{e}\left(x_{e}\right)=\alpha_{e}, \quad \forall e \in \mathcal{E} .
$$

However, when $L \neq 0$ this technique cannot be applied since a priori no information is available for the boundary term in (4.1).

Exploiting the fact that $-\Delta \psi=0$, with $\psi \in H^{2}(\Gamma)$, implies

$$
\psi_{e}\left(x_{e}\right)= \begin{cases}\alpha_{e}+\beta_{e} x_{e}, & e \in \mathcal{E}_{\mathrm{int}}, \\ 0, & e \in \mathcal{E}_{\mathrm{ex}} .\end{cases}
$$

Conditions on the vectors $\boldsymbol{\alpha}=\left(\alpha_{e}\right)_{e \in \mathcal{E}_{\text {int }}}^{T}$ and $\boldsymbol{\beta}=\left(\beta_{e}\right)_{e \in \mathcal{E}_{\text {int }}}^{T}$ were derived in 19]. We shall give the same conditions in a slightly different form, on the 
vectors $(\boldsymbol{\alpha}, \boldsymbol{\beta}, 0)^{T} \in M \subset \mathbb{C}^{E}$, and for this purpose introduce the matrices

$$
C(\boldsymbol{l}):=\left(\begin{array}{ccc}
\mathbb{1}_{\mathcal{E}_{\mathrm{int}}} & 0 & 0 \\
\mathbb{1} & D(\boldsymbol{l}) & 0 \\
0 & 0 & 0_{\mathcal{E}_{\mathrm{ex}}}
\end{array}\right) \quad \text { and } \quad V:=\left(\begin{array}{ccc}
0 \mathcal{E}_{\mathrm{int}} & \mathbb{1} & 0 \\
0 & -\mathbb{1}_{\mathcal{E}_{\mathrm{int}}} & 0 \\
0 & 0 & 0 \\
\mathcal{E}_{\mathrm{ex}}
\end{array}\right) \text {. }
$$

Lemma 4.1. The vector $(\boldsymbol{\alpha}, \boldsymbol{\beta}, 0)^{T} \in M$ contains the coefficients of a zeromode, iff $\boldsymbol{v}:=C(\boldsymbol{l})\left(\boldsymbol{\alpha}, \boldsymbol{\beta}, \mathbf{0}_{\mathcal{E}_{\text {ex }}}\right)^{T} \in M$ satisfies

$$
\begin{aligned}
& \mathbf{0}=P_{\operatorname{ran} L}\left(L_{\mathrm{MBP}}^{-1} G(\boldsymbol{l})-\mathbb{1}_{\mathbb{C}^{E}}\right) \boldsymbol{v}, \\
& \mathbf{0}=P^{\perp} \boldsymbol{v}-\boldsymbol{v}, \\
& \mathbf{0}=Q G(\boldsymbol{l}) \boldsymbol{v}-G(\boldsymbol{l}) \boldsymbol{v} .
\end{aligned}
$$

Proof. The vertex conditions are

$$
\begin{aligned}
\mathbf{0} & =(P+L)(\boldsymbol{\alpha}, \boldsymbol{\alpha}+D(\boldsymbol{l}) \boldsymbol{\beta}, \mathbf{0})^{T}+P^{\perp} I(\boldsymbol{\beta}, \boldsymbol{\beta}, \mathbf{0})^{T} \\
& =P \boldsymbol{v}+\left(L C(\boldsymbol{l})+P^{\perp} V\right)(\boldsymbol{\alpha}, \boldsymbol{\beta}, \mathbf{0})^{T} .
\end{aligned}
$$

Due to orthogonality, the first and the second term in the second line vanish separately. Hence the second line in (4.5) follows. Moreover,

$$
L C(\boldsymbol{l})+P^{\perp} V=\left(L C(\boldsymbol{l})+P_{\operatorname{ran} L} V\right)-(Q V-V),
$$

where on the right-hand side the terms in the brackets are orthogonal with respect to each other. Hence,

$$
\begin{aligned}
& \mathbf{0}=P_{\mathrm{ran} L} V\left(\boldsymbol{\alpha}, \boldsymbol{\beta}, \mathbf{0}_{\mathcal{E}_{\mathrm{ex}}}\right)^{T}+L C(\boldsymbol{l})\left(\boldsymbol{\alpha}, \boldsymbol{\beta}, \mathbf{0}_{\mathcal{E}_{\mathrm{ex}}}\right)^{T}, \\
& \mathbf{0}=Q V\left(\boldsymbol{\alpha}, \boldsymbol{\beta}, \mathbf{0}_{\mathcal{E}_{\mathrm{ex}}}\right)^{T}-V\left(\boldsymbol{\alpha}, \boldsymbol{\beta}, \mathbf{0}_{\mathcal{E}_{\mathrm{ex}}}\right)^{T} .
\end{aligned}
$$

On $M$ the matrix $C(\boldsymbol{l})$ is invertible and its $M$-inverse coincides with

$$
C(\boldsymbol{l})_{\mathrm{MBP}}^{-1}=\left(\begin{array}{ccc}
\mathbb{1}_{\mathcal{E}_{\text {int }}} & 0 & 0 \\
D(\boldsymbol{l})^{-1} & D(\boldsymbol{l})^{-1} & 0 \\
0 & 0 & 0_{\mathcal{E}_{\mathrm{ex}}}
\end{array}\right)
$$

implying

$$
G(\boldsymbol{l})=-V C(\boldsymbol{l})_{\mathrm{MBP}}^{-1}
$$

Moreover,

$$
L_{\mathrm{MBP}}^{-1} L=L L_{\mathrm{MBP}}^{-1}=P_{\operatorname{ran} L},
$$

so that (4.8) implies the first and the third line of (4.5).

In view of the first equation in (4.5) we need to invert $L_{\mathrm{MBP}}^{-1} G(\boldsymbol{l})-\mathbb{1}_{\mathbb{C}^{E}}$ on $\operatorname{ran} L$. This can be done when, e.g., 1 is not an eigenvalue of $L_{\mathrm{MBP}}^{-1} G(\boldsymbol{l})$. According to Lemma 3.11 this will be the case if one assumes that $l_{\min }>$ $2 / \lambda_{\text {min }}^{+}$, and hence the largest eigenvalue of $L_{\mathrm{MBP}}^{-1} G(\boldsymbol{l})$ satisfies $\tau_{\max }<1$. In such a case $\left(L_{\mathrm{MBP}}^{-1} G(\boldsymbol{l})-\mathbb{1}_{\mathbb{C}^{E}}\right)^{-1}$ exists.

We are now in a position to formulate our characterisation of the zero modes. 
Theorem 4.2. Let $-\Delta_{P, L}$ be an arbitrary self-adjoint realisation of the Laplacian on a metric graph $\Gamma$ and assume that the maximal eigenvalue of $L_{\mathrm{MBP}}^{-1} G(\boldsymbol{l})$ satisfies $\tau_{\max }<1$. Then the coefficients $\boldsymbol{\alpha}=\left(\alpha_{e}\right)_{e \in \mathcal{E}_{\text {int }}}^{T}$ and $\boldsymbol{\beta}=\left(\beta_{e}\right)_{e \in \mathcal{E}_{\text {int }}}^{T}$ of the zero modes (4.3) are of the form

$$
\left(\boldsymbol{\alpha}, \mathbf{0}_{\mathcal{E}_{\text {int }}}, \mathbf{0}_{\mathcal{E}_{\text {ex }}}\right)^{T} \in\left(\begin{array}{cc}
\mathbb{1}_{\left|\mathcal{E}_{\text {int }}\right|} & 0 \\
0 & 0_{\mathcal{E}_{\text {int }} \oplus \mathcal{E}_{\text {ex }}}
\end{array}\right)\left(\operatorname{ker} Q \cap M_{\text {sy }}\right) .
$$

In particular, $\boldsymbol{\beta}=\mathbf{0}$ so that all zero modes are edge-wise constant. Furthermore, the spectral multiplicity $g_{0}$ of the Laplace eigenvalue zero is

$$
g_{0}=\operatorname{dim}\left(\operatorname{ker} Q \cap M_{\mathrm{sy}}\right) .
$$

Proof. We recall from Lemma 4.1 that $\boldsymbol{v}=C(\boldsymbol{l})\left(\boldsymbol{\alpha}, \boldsymbol{\beta}, \mathbf{0}_{\mathcal{E}_{\text {ex }}}\right)^{T} \in M$ has to satisfy three equations in order for $\boldsymbol{\alpha}$ and $\boldsymbol{\beta}$ to be related to a zero mode. The space of solutions $\boldsymbol{v}$ of the first equation is

$$
\mathfrak{V}_{1}:=\left.\operatorname{ran}\left(L_{\mathrm{MBP}}^{-1} G(\boldsymbol{l})-\mathbb{1}_{\mathbb{C}^{E}}\right)^{-1}\right|_{\operatorname{ker} L} .
$$

The case $\operatorname{ker} L=\emptyset$ implies $\mathfrak{V}_{1}=\emptyset$. Hence we consider $\operatorname{ker} L \neq \emptyset$. Under the assumption $\tau_{\max }<1$ the matrix $\left(L_{\mathrm{MBP}}^{-1} G(\boldsymbol{l})-\mathbb{1}_{\mathbb{C} E}\right)$ is invertible, and rearranging its Cayley-Hamilton polynomial gives

$$
\begin{aligned}
\left(L_{\mathrm{MBP}}^{-1} G(\boldsymbol{l})-\mathbb{1}_{\mathbb{C}^{E}}\right)^{-1} & =\sum_{n=0}^{E-1} a_{n}\left(L_{\mathrm{MBP}}^{-1} G(\boldsymbol{l})-\mathbb{1}_{\mathbb{C}^{E}}\right)^{n} \\
& =\sum_{n=0}^{E-1} b_{n}\left(L_{\mathrm{MBP}}^{-1} G(\boldsymbol{l})\right)^{n} \\
& =b_{0} \mathbb{1}_{\mathbb{C}^{E}}+L_{\mathrm{MBP}}^{-1} Z
\end{aligned}
$$

with complex coefficients $a_{n}, b_{n}$ and some matrix $Z \in \operatorname{Mat}(E \times E ; \mathbb{C})$. Choosing $\boldsymbol{w} \in \operatorname{ker} L \neq \emptyset$ one notices that

$$
\boldsymbol{v}=\left(L_{\mathrm{MBP}}^{-1} G(\boldsymbol{l})-\mathbb{1}_{\mathbb{C}^{E}}\right)^{-1} \boldsymbol{w} \quad \Leftrightarrow \quad\left(L_{\mathrm{MBP}}^{-1} G(\boldsymbol{l})-\mathbb{1}_{\mathbb{C}^{E}}\right) \boldsymbol{v}=\boldsymbol{w}
$$

requires $b_{0} \neq 0$. The second condition in Lemma 4.1 $P \boldsymbol{v}=0$, then implies via $\boldsymbol{v}=b_{0} \boldsymbol{w}+L_{\mathrm{MBP}}^{-1} Z \boldsymbol{w}$ that $P \boldsymbol{w}=\mathbf{0}$, hence $\boldsymbol{w} \in \operatorname{ker} P \cup \operatorname{ker} L=\operatorname{ker} Q$. Therefore, the space of solutions of the first and second equation in Lemma 4.1] is

$$
\mathfrak{V}_{1,2}=\left.\operatorname{ran}\left(L_{\mathrm{MBP}}^{-1} G(\boldsymbol{l})-\mathbb{1}_{\mathbb{C}^{E}}\right)^{-1}\right|_{\operatorname{ker} Q} .
$$

The third equation adds the condition

$$
G(\boldsymbol{l})\left(L_{\mathrm{MBP}}^{-1} G(\boldsymbol{l})-\mathbb{1}_{\mathbb{C}^{E}}\right)^{-1} \boldsymbol{w} \in(\operatorname{ker} Q)^{\perp} \quad \text { with } \quad \boldsymbol{w} \in \operatorname{ker} Q .
$$

In order to proceed from here we notice that

$$
G(\boldsymbol{l})\left(L_{\mathrm{MBP}}^{-1} G(\boldsymbol{l})-\mathbb{1}_{\mathbb{C}^{E}}\right)=\left(G(\boldsymbol{l}) L_{\mathrm{MBP}}^{-1}-\mathbb{1}_{\mathbb{C}^{E}}\right) G(\boldsymbol{l}),
$$

implies

$$
G(\boldsymbol{l})\left(L_{\mathrm{MBP}}^{-1} G(\boldsymbol{l})-\mathbb{1}_{\mathbb{C}^{E}}\right)^{-1}=\left(G(\boldsymbol{l}) L_{\mathrm{MBP}}^{-1}-\mathbb{1}_{\mathbb{C}^{E}}\right)^{-1} G(\boldsymbol{l}) .
$$


Hence, using that $L_{\mathrm{MBP}}^{-1}$ and $G(\boldsymbol{l})$ are hermitian, it follows that $G(\boldsymbol{l})\left(L_{\mathrm{MBP}}^{-1} G(\boldsymbol{l})-\mathbb{1}_{\mathbb{C}^{E}}\right)$ is hermitian too. Furthermore, with (4.22) and (4.20) we find that

$$
G(\boldsymbol{l})\left(L_{\mathrm{MBP}}^{-1} G(\boldsymbol{l})-\mathbb{1}_{\mathbb{C}^{E}}\right)^{-1}=P_{\text {asy }}\left(G(\boldsymbol{l}) L_{\mathrm{MBP}}^{-1}-\mathbb{1}_{\mathbb{C}^{E}}\right)^{-1} G(\boldsymbol{l}) P_{\text {asy }} .
$$

We now need an invertible regularisation of $G(\boldsymbol{l})$. For this we notice from its definition (3.50) that $G(\boldsymbol{l})$ is symmetric, non-negative, and that

$$
\operatorname{ker} G(\boldsymbol{l})=M_{\mathrm{sy}} \oplus M_{0}=M_{\mathrm{asy}}^{\perp} .
$$

This allows us to define

$$
G(\boldsymbol{l})_{\delta}:=G(\boldsymbol{l})+\delta^{2} P_{\mathrm{asy}}^{\perp}, \quad \delta>0,
$$

which is also symmetric and non-negative and, moreover, invertible. We then obtain

$$
G(\boldsymbol{l})\left(L_{\mathrm{MBP}}^{-1} G(\boldsymbol{l})-\mathbb{1}_{\mathbb{C}^{E}}\right)^{-1} G(\boldsymbol{l})_{\delta}{ }^{-1}=P_{\text {asy }}\left(G(\boldsymbol{l}) L_{\mathrm{MBP}}^{-1}-\mathbb{1}_{\mathbb{C}^{E}}\right)^{-1} P_{\text {asy }} .
$$

Following an extended version of Sylvester's inertia law [18, multiplying a hermitian matrix by a positive definite matrix leaves the number of positive and negative eigenvalues, respectively, unchanged. Therefore, the maps $G(\boldsymbol{l})\left(L_{\mathrm{MBP}}^{-1} G(\boldsymbol{l})-\mathbb{1}_{\mathbb{C}^{E}}\right)^{-1}$ and $P_{\text {asy }}\left(G(\boldsymbol{l}) L_{\mathrm{MBP}}^{-1}-\mathbb{1}_{\mathbb{C}^{E}}\right)^{-1} P_{\text {asy }}$ possess the same number of positive and negative eigenvalues. The non-zero spectrum of the latter is determined by

$$
P_{\text {asy }}\left(G(\boldsymbol{l}) L_{\mathrm{MBP}}^{-1}-\mathbb{1}_{\mathbb{C}^{E}}\right)^{-1} P_{\text {asy }} \boldsymbol{a}=\mu \boldsymbol{a}, \quad \text { with } \quad \boldsymbol{a} \in M_{\mathrm{asy}} .
$$

This is equivalent to

$$
\left(G(\boldsymbol{l}) L_{\mathrm{MBP}}^{-1}-\mathbb{1}_{\mathbb{C} E}\right) \boldsymbol{a}=\frac{1}{\mu} \boldsymbol{a}
$$

or

$$
G(\boldsymbol{l}) L_{\mathrm{MBP}}^{-1} \boldsymbol{a}=\left(L_{\mathrm{MBP}}^{-1} G(\boldsymbol{l})\right)^{*} \boldsymbol{a}=\left(\frac{1}{\mu}+1\right) \boldsymbol{a} .
$$

Lemma 3.11 therefore implies $\mu<0$ so that $P_{\text {asy }}\left(G(\boldsymbol{l}) L_{\mathrm{MBP}}^{-1}-\mathbb{1}_{\mathbb{C}^{E}}\right)^{-1} P_{\text {asy }}$, and by the inertia law also $G(\boldsymbol{l})\left(L_{\mathrm{MBP}}^{-1} G(\boldsymbol{l})-\mathbb{1}_{\mathbb{C}^{E}}\right)^{-1}$, is negative semi-definite. The negative of the latter hence has a hermitian, positive semi-definite square$\operatorname{root}\left(G(\boldsymbol{l})\left(\mathbb{1}_{\mathbb{C}^{E}}-L_{\mathrm{MBP}}^{-1} G(\boldsymbol{l})\right)^{-1}\right)^{-1 / 2}$. The condition (4.18) together with $\boldsymbol{w} \in \operatorname{ker} Q$ then yields

$$
\begin{aligned}
0=\mid\langle\boldsymbol{w}, G(\boldsymbol{l}) & \left.\left(L_{\mathrm{MBP}}^{-1} G(\boldsymbol{l})-\mathbb{1}_{\mathbb{C}^{E}}\right)^{-1} \boldsymbol{w}\right\rangle_{\mathbb{C}^{E}} \mid \\
= & \left\|\left(G(\boldsymbol{l})\left(\mathbb{1}_{\mathbb{C}^{E}}-L_{\mathrm{MBP}}^{-1} G(\boldsymbol{l})\right)^{-1}\right)^{1 / 2} \boldsymbol{w}\right\|_{\mathbb{C}^{E}},
\end{aligned}
$$

implying that

$$
\boldsymbol{w} \in \operatorname{ker}\left(G(\boldsymbol{l})\left(L_{\mathrm{MBP}}^{-1} G(\boldsymbol{l})-\mathbb{1}_{\mathbb{C}^{E}}\right)^{-1}\right) .
$$

Conversely, every $\boldsymbol{w}$ satisfying (4.29) also fulfils (4.18). Hence, the conditions (4.29) and $\boldsymbol{w} \in \operatorname{ker} Q$ are necessary and sufficient for $\boldsymbol{w}$ to satisfy (4.18). 
In order to finally prove (4.12) we use (4.29). From (4.25), the inertia law, and the fact that $\mu<0$ one concludes that

$$
\begin{aligned}
\operatorname{ker} & \left(G(\boldsymbol{l})\left(L_{\mathrm{MBP}}^{-1} G(\boldsymbol{l})-\mathbb{1}_{\mathbb{C}^{E}}\right)^{-1}\right) \\
& =\operatorname{ker}\left(P_{\text {asy }}\left(G(\boldsymbol{l}) L_{\mathrm{MBP}}^{-1}-\mathbb{1}_{\mathbb{C}^{E}}\right)^{-1} P_{\text {asy }}\right) \\
& =M_{\text {sy }} \oplus M_{0} .
\end{aligned}
$$

The third line follows by expanding $\left(G(\boldsymbol{l}) L_{\mathrm{MBP}}^{-1}-\mathbb{1}_{\mathbb{C}^{E}}\right)^{-1}$ in the same way as in (4.15), showing that this matrix leaves $M_{\text {asy }}$ invariant.

Hence, the conditions (4.29) and $\boldsymbol{w} \in \operatorname{ker} Q$ are equivalent to

$$
\boldsymbol{w} \in \operatorname{ker} Q \cap\left(M_{\text {sy }} \oplus M_{0}\right),
$$

which, together with the requirement that a zero-mode must vanish on the external edges, proves (4.12).

\section{The trace of the S-matrix and the zero-mode contribution in the trace formula}

The form (4.3) of zero modes is determined by the fact that apart from being a solution of $\Delta \psi=0$ one requires $\psi \in L^{2}(\Gamma)$, which determines that $\psi_{e}(x)=0$ when $e \in \mathcal{E}_{\text {ex }}$. For the following it turns out that a more general class of zero modes has to be considered.

Definition 5.1. A function $\phi \in C^{2}(\Gamma)$ is said to be a generalised zero mode, if $\Delta \phi=0$ and the boundary values $\underline{\phi}$ and $\phi^{\prime}$ satisfy the vertex conditions

$$
(P+L) \underline{\phi}+P^{\perp} I \underline{\phi^{\prime}}=0 .
$$

A generalised zero mode $\phi$ is said to be a proper generalised zero mode, if $\phi \notin L^{2}(\Gamma)$.

A zero mode is obviously a generalised zero mode too. A proper generalised zero mode, however, need not be edge-wise constant. A simple example for this is a single half-line with a Dirichlet vertex condition at the origin. Here the function $\psi(x)=x$ is a proper generalised zero mode. Edge-wise constant generalised zero modes will play a particular role.

Definition 5.2. We denote by $\mathfrak{G}_{0}$ the set of edge-wise constant generalised zero modes, and by $\mathfrak{G}_{p, 0} \subset \mathfrak{G}_{0}$ the subset of edge-wise constant proper generalised zero modes. We also set

$$
\widetilde{g}_{0}:=\operatorname{dim} \mathfrak{G}_{0} \quad \text { and } \quad \widetilde{g}_{p, 0}:=\operatorname{dim} \mathfrak{G}_{p, 0} .
$$

Note that Theorem 4.2 implies

$$
g_{0}=\widetilde{g}_{0}-\widetilde{g}_{p, 0} .
$$

In order to find expressions for the dimensions (5.2) we now associate a compact metric graph $\widehat{\Gamma}$ to a given (in general, non-compact) metric graph $\Gamma$. This construction is based on cutting every external edge $e \in \mathcal{E}_{\text {ex }}$ at some 
finite value and adding a vertex at the newly created edge end. More precisely, if $e \in \mathcal{E}_{\text {ex }}$, we replace this with an edge $\widehat{e}$ of length $l_{\widehat{e}}<\infty$ and add a vertex $v_{\widehat{e}}$ that is adjacent only to the edge $\widehat{e}$. We denote the sets of new edges and vertices by $\mathcal{E}_{\text {new }}$ and $\mathcal{V}_{\text {new }}$, respectively, both of cardinality $\left|\mathcal{E}_{\text {ex }}\right|$. This procedure defines a compact graph $\widehat{\Gamma}$ with edge set $\widehat{\mathcal{E}}=\mathcal{E}_{\text {int }} \cup \mathcal{E}_{\text {new }}$ and vertex set $\widehat{\mathcal{V}}=\mathcal{V} \cup \mathcal{V}_{\text {new }}$. There is an obvious, isometric embedding $\widehat{\Gamma} \rightarrow \Gamma$ of the compact graph into the original graph, provided by the identity map. This allows us to restrict a function $\psi$ on $\Gamma$ to $\widehat{\Gamma}$, and to identify the restriction $\widehat{\psi}:=\left.\psi\right|_{\widehat{\Gamma}}$ with a function on $\widehat{\Gamma}$. The latter has boundary values (if defined),

$$
\widehat{\psi}:=\left(\left\{\psi_{e}(0)\right\}_{e \in \mathcal{E}_{\mathrm{int}}},\left\{\psi_{e}\left(l_{e}\right)\right\}_{e \in \mathcal{E}_{\mathrm{int}}},\left\{\psi_{e}(0)\right\}_{e \in \mathcal{E}_{\text {new }}},\left\{\psi_{e}\left(l_{e}\right)\right\}_{e \in \mathcal{E}_{\text {new }}}\right)^{T},
$$

in $\mathbb{C}^{\widehat{E}}$ where $\widehat{E}=2\left(\left|\mathcal{E}_{\text {int }}\right|+\left|\mathcal{E}_{\text {ex }}\right|\right)$.

Given a self-adjoint realisation $-\Delta_{P, L}$ of the Laplacian on $\Gamma$, we associate with it operators $-\Delta_{\widehat{P}_{D / N}, \widehat{L}}$ on $\widehat{\Gamma}$ with Dirichlet- and Neumann-vertex conditions at the new vertices $v \in \mathcal{V}_{\text {new }}$, where

$$
\widehat{P}_{D / N}:=\left(\begin{array}{cc}
P & 0 \\
0 & \mathbb{1}_{\mathcal{E}_{\text {new }}} / 0_{\mathcal{E}_{\text {new }}}
\end{array}\right) \quad \text { and } \quad \widehat{L}:=\left(\begin{array}{cc}
L & 0 \\
0 & 0_{\mathcal{E}_{\text {new }}}
\end{array}\right) .
$$

Following Theorem 2.1, the operator $-\Delta_{\widehat{P}_{D / N}, \widehat{L}}$ is self-adjoint on the domain corresponding to (2.7). The associated $\mathfrak{S}$-matrix is,

$$
\mathfrak{S}_{\widehat{P}_{D / N}, \widehat{L}}(k)=\left(\begin{array}{cc}
\mathfrak{S}_{P, L}(k) & 0 \\
0 & \mp \mathbb{1}_{\mathcal{E}_{\text {new }}}
\end{array}\right), \quad k \notin \mathrm{i} \sigma(L) \backslash\{0\} .
$$

We then set $\widehat{Q}_{D / N}:=\widehat{P}_{D / N}+P_{\text {ran } \widehat{L}}$, as well as

$$
\begin{aligned}
\widehat{M}_{\mathrm{sy}} & :=\left\{\left(\boldsymbol{c}_{1}, \boldsymbol{c}_{1}, \boldsymbol{c}_{2}, \boldsymbol{c}_{2}\right)^{T} \in \mathbb{C}^{\widehat{E}} ; \quad \boldsymbol{c}_{1} \in \mathbb{C}^{\left|\mathcal{E}_{\text {int }}\right|}, \quad \boldsymbol{c}_{2} \in \mathbb{C}^{\left|\mathcal{E}_{\text {ex }}\right|}\right\}, \\
\widehat{M}_{\text {asy }} & :=\left\{\left(\boldsymbol{c}_{1},-\boldsymbol{c}_{1}, \boldsymbol{c}_{2},-\boldsymbol{c}_{2}\right)^{T} \in \mathbb{C}^{\widehat{E}} ; \quad \boldsymbol{c}_{1} \in \mathbb{C}^{\left|\mathcal{E}_{\text {int }}\right|}, \quad \boldsymbol{c}_{2} \in \mathbb{C}^{\left|\mathcal{E}_{\text {ex }}\right|}\right\} .
\end{aligned}
$$

We also define the spectral and algebraic multiplicities $\widehat{g}_{0 / N}$ and $\widehat{N}_{D / N}$, respectively of the eigenvalue zero of $\Delta_{\widehat{P}_{D / N}, \widehat{L}}$. Choosing the shortest additional length $l_{e}, e \in \mathcal{E}_{\text {new }}$ to be large enough, the maximal eigenvalue of $\widehat{L}_{\mathrm{MBP}}^{-1} G(\widehat{\boldsymbol{l}})$ satisfies $\widehat{\tau}_{\max }<1$, if $\tau_{\max }<1$.

Lemma 5.3. Assume that $\tau_{\max }<1$. Then

$$
{\widehat{g_{0}}}_{D}=g_{0}, \quad \widehat{g}_{0_{N}}=\widetilde{g}_{0},
$$

and

$$
\begin{aligned}
& \widehat{N}_{D}=N+\operatorname{dim}\left((\operatorname{ker} Q)^{\perp} \cap\left(M_{\text {asy }} \oplus M_{0}\right)\right)-\operatorname{dim}\left((\operatorname{ker} Q)^{\perp} \cap M_{\text {asy }}\right) \\
& \widehat{N}_{N}=\widetilde{g}_{0}+\operatorname{dim}\left((\operatorname{ker} Q)^{\perp} \cap M_{\text {asy }}\right) .
\end{aligned}
$$

Proof. As $\widehat{\Gamma}$ has only internal edges, $\psi \in \operatorname{ker} \Delta_{\widehat{P}_{D}, \widehat{L}}$ implies that $\psi_{e}(x)=\alpha_{e}$ for all $e \in \widehat{\mathcal{E}}$. The Dirichlet conditions imposed at every $v \in \mathcal{V}_{\text {new }}$ then imply that

$$
\psi_{e} \equiv 0 \text { for all } e \in \mathcal{E}_{\text {new }} \text {. }
$$


Hence, every zero mode of $-\Delta_{P, L}$ can be identified with a zero mode of $-\Delta_{\widehat{P}_{D}, \widehat{L}}$, and vice versa. This proves that ${\widehat{g_{0}}}_{D}=g_{0}$. Moreover, by (5.5),

$$
\begin{aligned}
\operatorname{ker} \widehat{Q}_{D} & =\left\{\left(\boldsymbol{v}, \mathbf{0}_{\mathcal{E}_{\text {ex }}}\right)^{T} \in \mathbb{C}^{\widehat{E}} ; \quad \boldsymbol{v} \in \operatorname{ker} Q\right\} \\
\left(\operatorname{ker} \widehat{Q}_{D}\right)^{\perp} & =\left\{(\boldsymbol{a}, \boldsymbol{d})^{T} \in \mathbb{C}^{\widehat{E}} ; \quad \boldsymbol{a} \in(\operatorname{ker} Q)^{\perp} \quad \boldsymbol{d} \in \mathbb{C}^{\left|\mathcal{E}_{\text {ex }}\right|}\right\} .
\end{aligned}
$$

Using (5.7) and (5.11) we then obtain

$$
\begin{array}{r}
\operatorname{dim}\left(\operatorname{ker} \widehat{Q}_{D} \cap \widehat{M}_{\mathrm{sy}}\right)=\operatorname{dim}\left(\operatorname{ker} Q \cap M_{\mathrm{sy}}\right), \\
\operatorname{dim}\left(\left(\operatorname{ker} \widehat{Q}_{D}\right)^{\perp} \cap \widehat{M}_{\text {asy }}\right)=\operatorname{dim}\left((\operatorname{ker} Q)^{\perp} \cap\left(M_{\text {asy }} \oplus M_{0}\right)\right) .
\end{array}
$$

Hence, Corollary 3.10 and Proposition 3.12 imply the first line of (5.9).

Similarly, for $\psi \in \operatorname{ker} \Delta_{\widehat{P}_{D}, \widehat{L}}$ the Neumann conditions at the new vertices, $v \in \mathcal{V}_{\text {new }}$, impose no restriction on $\psi_{e}$. Hence, such a $\psi$ can be extended to $\Gamma$ to yield an edge-wise constant generalised zero mode, and this process can be reversed. This implies that $\widehat{g}_{0 N}=\widetilde{g}_{0}$. Furthermore,

$$
\begin{aligned}
\operatorname{ker} \widehat{Q}_{N} & =\left\{(\boldsymbol{v}, \boldsymbol{d})^{T} \in \mathbb{C}^{\widehat{E}} ; \quad \boldsymbol{v} \in \operatorname{ker} Q, \quad \boldsymbol{d} \in \mathbb{C}^{E_{\text {ex }}}\right\}, \\
\left(\operatorname{ker} \widehat{Q}_{N}\right)^{\perp} & =\left\{\left(\boldsymbol{a}, \mathbf{0}_{\mathcal{E}_{\text {ex }}}\right)^{T} \in \mathbb{C}^{\widehat{E}} ; \quad \boldsymbol{a} \in(\operatorname{ker} Q)^{\perp}\right\} .
\end{aligned}
$$

With (5.11) and (5.13) this yields

$$
\begin{gathered}
\operatorname{dim}\left(\operatorname{ker} \widehat{Q}_{N} \cap \widehat{M}_{\mathrm{sy}}\right)=\operatorname{dim}\left((\operatorname{ker} Q) \cap\left(M_{\mathrm{sy}} \oplus M_{0}\right)\right), \\
\operatorname{dim}\left(\left(\operatorname{ker} \widehat{Q}_{N}\right)^{\perp} \cap \widehat{M}_{\text {asy }}\right)=\operatorname{dim}\left((\operatorname{ker} Q)^{\perp} \cap M_{\text {asy }}\right) .
\end{gathered}
$$

Corollary 3.10. Proposition 3.12 and (4.13) then imply the second line of (5.9).

As a consequence, any expression for the number of zero modes in terms of an $\mathfrak{S}$-matrix in the sense of (1.5) will require an expression for $\operatorname{tr} \mathfrak{S}_{Q}$ in terms of the various subspaces of $\mathbb{C}^{E}$ that occur above. Since (5.6) implies

$$
\operatorname{tr} \mathfrak{S}_{\widehat{P}_{D / N}, \widehat{L}}(k)=\operatorname{tr} \mathfrak{S}_{P, L}(k) \mp\left|\mathcal{E}_{\mathrm{ex}}\right|,
$$

instead of $\mathfrak{S}_{Q}$ one can work with

$$
\mathfrak{S}_{\widehat{Q}_{D / N}}:=\lim _{k \rightarrow 0} \mathfrak{S}_{\widehat{P}_{D / N}, \widehat{L}}(k)=\widehat{Q}_{D / N}^{\perp}-\widehat{Q}_{D / N} .
$$

This map, as well as $\mathfrak{J}_{\widehat{\mathcal{E}}}$, is related to the compact graph $\widehat{\Gamma}$. Hence, (3.8) implies that $\mathfrak{S}_{\widehat{Q}_{D / N}}$ and $\mathfrak{J}_{\widehat{\mathcal{E}}}$ are unitary and hermitian in $\mathbb{C}^{\widehat{E}}$ and, therefore, square to the identity. Thus $\mathfrak{S}_{\widehat{Q}} \mathfrak{J}_{\widehat{\mathcal{E}}}$ is unitary, with $\left(\mathfrak{S}_{\widehat{Q}} \mathfrak{J}_{\widehat{\mathcal{E}}}\right)^{-1}=\mathfrak{J}_{\widehat{\mathcal{E}}} \mathfrak{S}_{\widehat{Q}}$, and its eigenvalues lie on the unit circle in $\mathbb{C}$.

Definition 5.4. Let $\widehat{Q}$ be a projector in $\mathbb{C}^{\widehat{E}}$ and set $\mathfrak{S}_{\widehat{Q}}=\widehat{Q}^{\perp}-\widehat{Q}$. We then define the following sets: 
1. The set of all eigenvalues of $\mathfrak{S}_{\widehat{Q}} \mathfrak{J}_{\widehat{\mathcal{E}}}$ with positive (negative) imaginary part is denoted as $W^{+}\left(W^{-}\right)$.

2. Let $\left\{\boldsymbol{z}_{n}\right\}_{1 \leq n \leq l}$ be a maximal set of linearly independent eigenvectors corresponding to the eigenvalues $\lambda_{n} \in W^{+}$. Define

$$
\mathcal{W}^{(+, \pm)}:=\operatorname{lin}\left\{\left(\mathfrak{J}_{\widehat{\mathcal{E}}} \pm \lambda_{n}\right) \boldsymbol{z}_{n} ; \quad 1 \leq n \leq l\right\} .
$$

3. The sets $\mathcal{W}^{(-, \pm)}$corresponding to $\lambda_{n} \in W^{-}$are defined analogously.

These sets allow us to observe the following.

Lemma 5.5. Let $\widehat{Q}$ be a projector in $\mathbb{C}^{\widehat{E}}$, then $\boldsymbol{v}$ is an eigenvector of $\mathfrak{S}_{\widehat{Q}}$ with eigenvalue 1 , iff

$$
\boldsymbol{v} \in \mathcal{W}^{(+,+)} \oplus\left(\operatorname{ker} \widehat{Q} \cap \widehat{M}_{\mathrm{sy}}\right) \oplus\left(\operatorname{ker} \widehat{Q} \cap \widehat{M}_{\text {asy }}\right) .
$$

Furthermore, $\boldsymbol{a}$ is an eigenvector of $\mathfrak{S}_{\widehat{Q}}$ with eigenvalue -1 , iff

$$
\boldsymbol{a} \in \mathcal{W}^{(+,-)} \oplus\left((\operatorname{ker} \widehat{Q})^{\perp} \cap \widehat{M}_{\mathrm{sy}}\right) \oplus\left((\operatorname{ker} \widehat{Q})^{\perp} \cap \widehat{M}_{\text {asy }}\right) .
$$

Moreover, the relations

$$
\operatorname{dim} \mathcal{W}^{(+,+)}=\operatorname{dim} \mathcal{W}^{(+,-)}
$$

and

$$
\mathcal{W}^{(+,+)}=\mathcal{W}^{(-,+)}, \quad \mathcal{W}^{(+,-)}=\mathcal{W}^{(-,-)}
$$

hold.

Proof. Let $\boldsymbol{z} \in \mathbb{C}^{\widehat{E}}$ be an eigenvector of $\mathfrak{S}_{\widehat{Q}} \mathfrak{J}_{\widehat{\mathcal{E}}}$ with eigenvalue $\lambda$. Due to unitarity, $\lambda^{-1}=\bar{\lambda}$ and $\lambda^{-1} \boldsymbol{z}=\mathfrak{J}_{\widehat{\mathcal{E}}} \mathfrak{S}_{\widehat{Q}} \boldsymbol{z}$, so that $\mathfrak{S}_{\widehat{Q}} \boldsymbol{z}=\bar{\lambda}_{\mathfrak{J}_{\widehat{\mathcal{E}}}} \boldsymbol{z}$. Hence, first,

$$
\begin{aligned}
& \mathfrak{S}_{\widehat{Q}}\left(\mathfrak{J}_{\widehat{\mathcal{E}}} \boldsymbol{z}+\lambda \boldsymbol{z}\right)=\mathfrak{J}_{\widehat{\mathcal{E}}} \boldsymbol{z}+\lambda \boldsymbol{z}, \\
& \mathfrak{S}_{\widehat{Q}}\left(\mathfrak{J}_{\widehat{\mathcal{E}}} \boldsymbol{z}-\lambda \boldsymbol{z}\right)=-\left(\mathfrak{J}_{\widehat{\mathcal{E}}} \boldsymbol{z}-\lambda \boldsymbol{z}\right),
\end{aligned}
$$

and, furthermore,

$$
\mathfrak{S}_{\widehat{Q}} \mathfrak{J}_{\widehat{\mathcal{E}}} \mathfrak{J}_{\widehat{\mathcal{E}}} \boldsymbol{z}=\bar{\lambda} \mathfrak{J}_{\widehat{\mathcal{E}}} \boldsymbol{z}
$$

First consider $\lambda \in W^{ \pm}$, i.e., $\lambda \neq \pm 1$. The eigenspaces corresponding to such a $\lambda$ and to $\bar{\lambda} \neq \lambda$, respectively, are orthogonal. Hence, by (5.23), the eigenvectors $\boldsymbol{z}$ and $\mathfrak{J}_{\widehat{\mathcal{E}}} \boldsymbol{z}$ of $\mathfrak{S}_{\widehat{Q}} \mathfrak{J}_{\widehat{\mathcal{E}}}$ are orthogonal. Thus the vectors $\mathfrak{J}_{\widehat{\mathcal{E}}} \boldsymbol{z} \pm \lambda \boldsymbol{z}$ are non-zero and, therefore, are eigenvectors of $\mathfrak{S}_{\widehat{Q}}$ with eigenvalues \pm 1 , respectively. Hence $\mathcal{W}^{( \pm,+)}$are subspaces of the $\mathfrak{S}_{\widehat{Q}^{-}}$-eigenspace with eigenvalue 1 , and $\mathcal{W}^{( \pm,-)}$are subspaces of the $\mathfrak{S}_{\widehat{Q}^{-}}$-eigenspace with eigenvalue -1 , yielding the first direct summands on the right-hand sides of (5.18) and (5.19), respectively. Due to the unitarity of $\mathfrak{S}_{\widehat{Q}} \mathfrak{J}_{\widehat{\mathcal{E}}}$ the cardinalities of $W^{+}$and $W^{-}$ (counted with multiplicities) coincide, and also coincide with the dimensions (5.20).

Multiplying both equations in (5.22) with $\bar{\lambda}$ we observe that the eigenvectors of $\mathfrak{S}_{\widehat{Q}}$ corresponding to the eigenvalues \pm 1 constructed from $\boldsymbol{z}$ and from $\mathfrak{J}_{\widehat{\mathcal{E}}} \boldsymbol{z}$ are proportional. This implies the relations (5.21). 
Now consider $\lambda= \pm 1$, hence Lemma 3.9 applies. For this reason we treat $\boldsymbol{z} \in \widehat{M}_{\mathrm{sy}}$ and $\boldsymbol{z} \in \widehat{M}_{\text {asy }}$ separately. Notice that (3.19) implies $\mathfrak{J}_{\widehat{\mathcal{E}}} \boldsymbol{z}=\boldsymbol{z}$ for $\boldsymbol{z} \in$ $\widehat{M}_{\text {sy }}$ and $\mathfrak{J}_{\widehat{\mathcal{E}}} \boldsymbol{z}=-\boldsymbol{z}$ for $\boldsymbol{z} \in \widehat{M}_{\text {asy }}$. Such vectors are automatically eigenvectors of $\mathfrak{S}_{\widehat{Q}}$ with eigenvalues \pm 1 . In two of the four cases, corresponding to $\lambda=$ \pm 1 and $\boldsymbol{z} \in \widehat{M}_{\mathrm{sy} / \text { asy }}$, the eigenvectors $\mathfrak{J}_{\widehat{\mathcal{E}}} \boldsymbol{z} \pm \lambda \boldsymbol{z}$ of $\mathfrak{S}_{\widehat{Q}}$ vanish, and in the remaining cases they are $\pm 2 \boldsymbol{z}$. Hence, $\boldsymbol{z}$ is an eigenvector of $\mathfrak{S}_{\widehat{Q}}$. Therefore, the dimensions of the direct sum of the eigenspaces of $\mathfrak{S}_{\widehat{Q}} \mathfrak{J}_{\widehat{\mathcal{E}}}$ corresponding to $\lambda= \pm 1$ and of the eigenspaces of $\mathfrak{S}_{\widehat{Q}}$ for the eigenvalues \pm 1 (via (5.22) ) are the same. Via Lemma 3.9 this finalises the proof of the identities given in (5.18) and (5.19).

We are now in a position to determine the trace of $\mathfrak{S}_{\widehat{Q}}$.

Proposition 5.6. Let $\widehat{Q}$ be an orthogonal projector in $\mathbb{C}^{\widehat{E}}$. Then,

$$
\begin{aligned}
\operatorname{tr} \mathfrak{S}_{\widehat{Q}} & =2\left[\operatorname{dim}\left(\operatorname{ker} \widehat{Q} \cap \widehat{M}_{\text {sy }}\right)-\operatorname{dim}\left((\operatorname{ker} \widehat{Q})^{\perp} \cap \widehat{M}_{\text {asy }}\right)\right] \\
& =2\left[\operatorname{dim}\left(\operatorname{ker} \widehat{Q} \cap \widehat{M}_{\text {asy }}\right)-\operatorname{dim}\left((\operatorname{ker} \widehat{Q})^{\perp} \cap \widehat{M}_{\text {sy }}\right)\right] .
\end{aligned}
$$

Proof. We first show the second equality in (5.24), and assume that

$$
(\operatorname{ker} \widehat{Q})^{\perp} \cap \widehat{M}_{\text {sy } / \text { asy }} \neq\{\mathbf{0}\}, \quad \operatorname{ker} \widehat{Q} \cap \widehat{M}_{\text {sy } / \text { asy }} \neq\{\mathbf{0}\},
$$

and

$$
\operatorname{dim} \mathcal{W}^{(+,+)}=\operatorname{dim} \mathcal{W}^{(+,-)} \neq 0 .
$$

All other cases can be treated analogously. We define

$$
\begin{aligned}
& M_{1}:=\mathbb{C}^{E} \ominus\left(\left((\operatorname{ker} \widehat{Q})^{\perp} \cap \widehat{M}_{\text {asy }}\right) \oplus\left(\operatorname{ker} \widehat{Q} \cap \widehat{M}_{\text {asy }}\right)\right), \\
& M_{2}:=\mathbb{C}^{E} \ominus\left(\left((\operatorname{ker} \widehat{Q})^{\perp} \cap \widehat{M}_{\mathrm{sy}}\right) \oplus\left(\operatorname{ker} \widehat{Q} \cap \widehat{M}_{\mathrm{sy}}\right)\right) .
\end{aligned}
$$

From Lemma 5.5 we then deduce that

$$
M_{1} \oplus M_{2}=\mathcal{W}^{(+,+)} \oplus \mathcal{W}^{(+,-)}
$$

holds. Let us assume that

$$
M_{1} \cap \mathcal{W}^{(+,+)} \neq\{0\},
$$

and choose $\mathbf{0} \neq \boldsymbol{b} \in M_{1} \cap \mathcal{W}^{(+,+)}$. We recall that all elements of $\mathcal{W}^{(+,+)}$ are eigenvectors of $\mathfrak{S}_{\widehat{Q}}$ corresponding to the eigenvalue 1 and therefore are elements of $\operatorname{ker} \widehat{Q}$. By construction we infer

$$
\boldsymbol{b} \in \operatorname{ker} \widehat{Q} \cap \widehat{M}_{\mathrm{asy}},
$$

which leads to a contradiction to $\boldsymbol{b} \in M_{1}$. Thus, we obtain

$$
M_{1} \cap \mathcal{W}^{(+,+)}=\{0\} .
$$

Similar arguments lead to

$$
M_{1} \cap \mathcal{W}^{(+,-)}=\{0\}, \quad M_{2} \cap \mathcal{W}^{(+,+)}=\{0\}, \quad M_{2} \cap \mathcal{W}^{(+,-)}=\{0\} .
$$


Therefore, every element of $\mathcal{W}^{(+,+)}$and $\mathcal{W}^{(+,-)}$is an orthonormal sum of non-zero vectors in $M_{1}$ and $M_{2}$. Eq. (5.20) hence implies

$$
\begin{aligned}
\operatorname{dim}\left((\operatorname{ker} \widehat{Q})^{\perp} \cap \widehat{M}_{\text {asy }}\right)+\operatorname{dim}\left(\operatorname{ker} \widehat{Q} \cap \widehat{M}_{\text {asy }}\right) & \\
= & \operatorname{dim}\left((\operatorname{ker} \widehat{Q})^{\perp} \cap \widehat{M}_{\text {sy }}\right)+\operatorname{dim}\left(\operatorname{ker} \widehat{Q} \cap \widehat{M}_{\text {sy }}\right) .
\end{aligned}
$$

Due to Lemma 5.5]

$$
\begin{aligned}
\operatorname{tr} \mathfrak{S}_{\widehat{Q}}=\operatorname{dim} & \left(\operatorname{ker} \widehat{Q} \cap \widehat{M}_{\mathrm{sy}}\right)+\operatorname{dim}\left(\operatorname{ker} \widehat{Q} \cap \widehat{M}_{\text {asy }}\right) \\
& -\operatorname{dim}\left((\operatorname{ker} \widehat{Q})^{\perp} \cap \widehat{M}_{\mathrm{sy}}\right)-\operatorname{dim}\left((\operatorname{ker} \widehat{Q})^{\perp} \cap \widehat{M}_{\text {asy }}\right) .
\end{aligned}
$$

Combining this with (5.33) gives the claim.

Now we can present our final result for the trace of the $\mathfrak{S}$-matrix.

Theorem 5.7. Given an arbitrary self-adjoint realisation $\Delta_{P, L}$ of the Laplacian on a metric graph with spectral and algebraic multiplicities $g_{0}$ and $N$ of the eigenvalue zero, respectively. Furthermore, assume that the maximal eigenvalue of $L_{\mathrm{MBP}}^{-1} G(\boldsymbol{l})$ satisfies $\tau_{\max }<1$. Then

$$
g_{0}-\frac{N}{2}=\frac{1}{4} \operatorname{tr} \mathfrak{S}_{0}+\frac{\left|\mathcal{E}_{\mathrm{ex}}\right|}{4}-\frac{\widehat{g}_{p, 0}}{2}
$$

holds, where $\widehat{g}_{p, 0}$ is the dimension of the space of edgewise constant proper generalised zero modes.

Proof. Applying Corollary 3.10 and Theorem 4.2 to $-\Delta_{\widehat{P}_{D}, \widehat{L}}$ gives

$$
2{\widehat{g_{0}}}_{D}-\widehat{N}_{D}=\frac{\operatorname{tr} \mathfrak{S}_{0}\left(\widehat{P}_{D}, \widehat{L}\right)}{2}=\frac{\operatorname{tr} \mathfrak{S}_{0}}{2}-\frac{\left|\mathcal{E}_{\mathrm{ex}}\right|}{2}
$$

where the second equality follows from (5.6). On the other hand, Lemma 5.3 yields

$$
\begin{aligned}
2{\widehat{g_{0}}}_{D} & -\widehat{N}_{D}=2 g_{0}-N \\
& -\left(\operatorname{dim}\left((\operatorname{ker} Q)^{\perp} \cap\left(M_{\text {asy }} \oplus M_{0}\right)\right)+\operatorname{dim}\left((\operatorname{ker} Q)^{\perp} \cap M_{\text {asy }}\right)\right) .
\end{aligned}
$$

In order to calculate the expression in the last line we use Proposition 5.6 to obtain

$$
\begin{aligned}
\operatorname{tr} \mathfrak{S}_{0} & \left(\widehat{P}_{N}, \widehat{L}\right)-\operatorname{tr} \mathfrak{S}_{0}\left(\widehat{P}_{D}, \widehat{L}\right) \\
= & 2\left[\operatorname{dim}\left((\operatorname{ker} Q)^{\perp} \cap\left(M_{\text {asy }} \oplus M_{0}\right)\right)-\operatorname{dim}\left((\operatorname{ker} Q)^{\perp} \cap M_{\text {asy }}\right)\right] \\
& -2\left[\operatorname{dim}\left(\operatorname{ker} Q \cap M_{\text {sy }}\right)-\operatorname{dim}\left(\operatorname{ker} Q \cap\left(M_{\text {sy }} \oplus M_{0}\right)\right)\right] .
\end{aligned}
$$

On the other hand, by (5.6) we find that

$$
\operatorname{tr} \mathfrak{S}_{0}\left(\widehat{P}_{N}, \widehat{L}\right)-\operatorname{tr} \mathfrak{S}_{0}\left(\widehat{P}_{D}, \widehat{L}\right)=2\left|\mathcal{E}_{\mathrm{ex}}\right|
$$


Therefore, using Theorem 4.2 together with (5.14) gives

$$
\begin{aligned}
\operatorname{dim} & \left((\operatorname{ker} Q)^{\perp} \cap\left(M_{\text {asy }} \oplus M_{0}\right)\right)-\operatorname{dim}\left((\operatorname{ker} Q)^{\perp} \cap M_{\text {asy }}\right) \\
& =\left|\mathcal{E}_{\text {ex }}\right|+g_{0}-\widehat{g}_{0, N} .
\end{aligned}
$$

Combining (5.36), (5.37) and (5.40) then proves the claim.

In the case of a compact graph $\Gamma$, where $\mathcal{E}_{\mathrm{ex}}=\emptyset$ and there are no proper generalised zero modes, the left-hand side of (5.35) is the constant $\gamma$ appearing in the zero-mode contribution to the trace formula (1.6), compare (3.13).

Corollary 5.8. In the situation of Theorem 5.7 assume that $\Gamma$ is a compact metric graph. Then

$$
\gamma=g_{0}-\frac{N}{2}=\frac{1}{4} \operatorname{tr} \mathfrak{S}_{0}
$$

This is an extension of the result in [10] where the case of non-Robin vertex conditions, corresponding to $L=0$, was treated. In that case $\mathfrak{S}_{P, L}(k)$ is independent of $k$.

\section{Factorisation of a non-positive Laplacian: a simple example}

An interval is the simplest example of a metric graph. For this case we first construct a Dirac operator in the sense of [11 along with a factorisation of the associated Laplacian in the form $-\Delta=p p^{*}$, where $p$ is a suitable 'momentum' operator. We then modify the Laplacian in order to generate a negative spectrum and discuss the necessary modifications in order to preserve the factorisation.

The metric graph $\Gamma$ has one edge that is identified with the interval $(0, l)$, and vertices $\mathcal{V}=\left\{v_{1}, v_{2}\right\}$ at the edge ends.

In this case the 0 -form space is $L^{2}(\Gamma)=L^{2}(0, l)$ and the 1 -form space is $L^{2}(0, l) \oplus \mathbb{C}^{2}$. The Dirac operator $D$ is defined in the Hilbert space

$$
\mathfrak{H}_{\Gamma}=L^{2}(0, l) \oplus\left(L^{2}(0, l) \oplus \mathbb{C}^{2}\right) .
$$

We require the associated Laplacian to be local and hence we introduce the linear maps

$$
P=\left(\begin{array}{cc}
P_{1} & 0 \\
0 & P_{2}
\end{array}\right) \quad \text { and } \quad L=\left(\begin{array}{cc}
L_{1} & 0 \\
0 & L_{2}
\end{array}\right),
$$

on $\mathbb{C}^{2}$, where $P_{j} \in\{0,1\}$. Furthermore, we require $L_{j} \leq 0$ and, in particular, $L_{j}=0$ if $P_{j}=1$. This ensures that $L$ is defined on $\operatorname{ker} P=\operatorname{ran} P^{\perp}$. The map (2.3) takes the form

$$
I=\left(\begin{array}{cc}
1 & 0 \\
0 & -1
\end{array}\right)
$$

Next we define the operators $d: \mathcal{D}_{d} \rightarrow L^{2}(0, l) \oplus \mathbb{C}^{2}$ and $d^{*}: \mathcal{D}_{d^{*}} \rightarrow L^{2}(0, l)$. On the domain

$$
\mathcal{D}_{d}:=\left\{\phi \in H^{1}(0, l) ; \quad P \underline{\phi}=0\right\},
$$


we set

$$
d \phi:=\left(-\phi^{\prime}, \sqrt{-L} \phi\right)
$$

Hence, the adjoint operator is defined on

$$
\mathcal{D}_{d^{*}}:=\left\{(\psi, \boldsymbol{a}) \in H^{1}(0, l) \oplus \mathbb{C}^{2} ; \quad P^{\perp} I \underline{\psi}+\sqrt{-L} \boldsymbol{a}=0\right\}
$$

to act as

$$
d^{*}(\psi, \boldsymbol{a}):=\psi^{\prime} .
$$

This then implies that the operator $d^{*} d$ is defined on the domain

$$
\mathcal{D}_{d^{*} d}=\left\{\psi \in H^{2}(0, l) ; \quad P \underline{\psi}=0, \quad P^{\perp} I \underline{\psi}^{\prime}+L \underline{\psi}=0\right\}
$$

and acts as

$$
d^{*} d \psi=-\psi^{\prime \prime} \text {. }
$$

One immediately recognises a self-adjoint realisation of the Laplacian, $d^{*} d=$ $-\Delta_{P, L}$, as described in Theorem 2.1

Remark 6.1. At a vertex $v_{j}$ where $P_{j}=1$ the vertex conditions in (6.4) and (6.8) enforce a Dirichlet condition. When $P_{j}=0$ and $L_{j}<0$, however, a Robin vertex condition is imposed. This can be seen as a repulsive $\delta$-potential of strength $\lambda_{j}=-2 L_{j}>0$ located at the vertex $v_{j}$ (cf. [3, 20]).

Apart from locality, the only restriction imposed in the example above was for $L$ to be negative semi-definite. This was necessary in the factorisation of the Laplacian due to the square roots in (6.6) and (6.5). Hence, whenever an attractive $\delta$-potential were to be introduced in a vertex, the associated Laplacian could no longer be factorised (see also [10, Section 6]). This observation coincides with the fact that a Laplacian with an attractive $\delta$-potential has negative spectrum.

An alternative factorisation of the Laplacian with an attractive $\delta$-potential can be guessed from the above example. As one must avoid square roots of $-L$, one could be tempted to remove the square root in (6.6) and, instead, replace the square root in (6.5) by a factor $-L$. Formally, (6.8) and (6.9) would still hold, but the operator $\left(d^{*}, \mathcal{D}_{d^{*}}\right)$ would no longer be the adjoint of $\left(d, \mathcal{D}_{d}\right)$ in the given Hilbert space setting. To remedy this problem one could modify the inner product in such a way that the strengths $\lambda_{j}$ of the $\delta$ potentials are included in its $\mathbb{C}^{2}$-contribution. As the $\lambda_{j}$ 's are not necessarily positive, however, this modification would no longer be an inner product.

Guided by this observation we conclude that replacing the Hilbert space structure by a Kreĭn space structure may provide a way to factorise any self-adjoint realisation of a Laplacian on a graph. Assuming that $L_{j} \neq 0$, $j=1,2$, in the example above, we therefore now introduce the non-degenerate hermitian form

$$
\mathfrak{a}_{L}((\phi, \boldsymbol{a}),(\psi, \boldsymbol{b})):=\langle\phi, \psi\rangle_{L^{2}(0, l)}+\left\langle\boldsymbol{a}, L^{-1} \boldsymbol{b}\right\rangle_{\mathbb{C}^{2}}
$$

on the one-form space $L^{2}(0, l) \oplus \mathbb{C}^{2}$ (viewed as a vector space). This form is used to define the non-degenerate hermitian form

$$
\mathfrak{A}_{L}((\varphi,(\phi, \boldsymbol{a})),(\xi,(\psi, \boldsymbol{b})))=\langle\varphi, \xi\rangle_{L^{2}(0, l)}+\mathfrak{a}_{L}((\phi, \boldsymbol{a}),(\psi, \boldsymbol{b}))
$$


on the vector space $\mathfrak{H}_{\Gamma}=L^{2}(0, l) \oplus\left(L^{2}(0, l) \oplus \mathbb{C}^{2}\right)$ (cf. 6.1) $)$. Equipped with the form (6.11), this vector space is a Kreïn space (see Section 7 below for more details).

For later purposes we now introduce momentum-like operators instead of the differential-like quantities (6.7) and (6.5). On the domain

$$
\mathcal{D}_{p}:=\left\{(\psi, \boldsymbol{a}) \in H^{1}(0, l) \oplus \mathbb{C}^{2} ; \quad P^{\perp} I \underline{\psi}-\mathrm{i} P_{\operatorname{ran} L} \boldsymbol{a}=0\right\},
$$

we define $p: \mathcal{D}_{p} \rightarrow L^{2}(0, l)$ to act as

$$
p(\psi, \boldsymbol{a}):=-\mathrm{i} \psi^{\prime} .
$$

An adjoint operator $p^{*}: \mathcal{D}_{p^{*}} \rightarrow L^{2}(0, l) \oplus \mathbb{C}^{2}$ with respect to the hermitian form (6.11) can be defined in close analogy to the Hilbert space setting in a more or less obvious way (for details see Section 7). It is defined on the domain

$$
\mathcal{D}_{p^{*}}:=\left\{\phi \in H^{1}(0, l) ; \quad P \underline{\phi}=0\right\}
$$

as

$$
p^{*} \phi:=\left(-\mathrm{i} \phi^{\prime},-L \underline{\phi}\right) .
$$

We now define the Dirac operator

$$
D:=\left(\begin{array}{cc}
0 & p \\
p^{*} & 0
\end{array}\right)
$$

on the domain

$$
\mathcal{D}_{D}=\mathcal{D}_{p^{*}} \oplus \mathcal{D}_{p}
$$

and observe that this is self-adjoint with respect to the form (6.11). Moreover, the operator $p p^{*}$ on the domain

$$
\mathcal{D}_{p p^{*}}=\left\{\psi \in H^{2}(0, l) ; \quad P^{\perp} I \underline{\psi}^{\prime}+(P+L) \underline{\psi}=0\right\}
$$

can be seen to be the self-adjoint realisation of the Laplacian described in Theorem 2.1. Note that here $L$ is no longer required to be negative semidefinite. Below we shall extend this construction to general graphs and general self-adjoint vertex conditions, and this will include the possibility of a zero eigenvalue of $L$.

\section{Kreĭn spaces and momentum operators}

In order to prepare for the construction of Dirac operators in Krel̆n spaces we first recall some basic facts on Krel̆n spaces and operators in such spaces, see [21] for details.

Definition 7.1. - A pair $(\mathfrak{K},[\cdot, \cdot])$, where $\mathfrak{K}$ is a complex vector space and $[\cdot, \cdot]$ is a hermitian form, is said to be an indefinite-metric vector space.

- Let $(\mathfrak{K},[\cdot, \cdot])$ be an indefinite-metric vector space and let $M \subset \mathfrak{K}$ be a subspace. Then the orthogonal complement $M^{\perp}$ is defined as

$$
M^{\perp}:=\{v \in \mathfrak{K} ; \quad[v, m]=0, \quad \forall m \in M\} .
$$


- A Kreı̆n space is an indefinite-metric vector space admitting a $[\cdot, \cdot]$ orthogonal decomposition

$$
\mathfrak{K}=\mathfrak{K}_{+} \oplus \mathfrak{K}_{-}, \quad \mathfrak{K}_{ \pm}{ }^{\perp}=\mathfrak{K}_{\mp},
$$

such that $\left(\mathfrak{K}_{+},[\cdot, \cdot]\right)$ and $\left(\mathfrak{K}_{-},-[\cdot, \cdot]\right)$ are Hilbert spaces.

- For a fixed decomposition (7.2) we associate to the KreĬn space $(\mathfrak{K},[\cdot, \cdot])$ a Hilbert space $\left(\mathfrak{K},(\cdot, \cdot)_{[\cdot, \cdot]}\right)$ by setting

$$
(x, y)_{[\cdot, \cdot]}:=\left[x_{+}, y_{+}\right]-\left[x_{-}, y_{-}\right],
$$

where $x=x_{+}+x_{-}$and $y=y_{+}+y_{-}$with $x_{ \pm}, y_{ \pm} \in \mathfrak{K}_{ \pm}$.

Lemma 7.2 (cf. [21]). Let $\left(\mathfrak{K}_{j} ;[\cdot, \cdot]_{j}\right), j=1,2$, be Kreĭn spaces and let $\mathcal{O}$ : $\mathcal{D}_{\mathcal{O}} \rightarrow \mathfrak{K}_{2}$ be an operator defined on a dense domain $\mathcal{D}_{\mathcal{O}} \subset \mathfrak{K}_{1}$. Then there exists a dense subspace $\mathcal{D}_{\mathcal{O}^{*}} \subset \mathfrak{K}_{2}$ and an operator $\mathcal{O}^{*}: \mathcal{D}_{\mathcal{O}^{*}} \rightarrow \mathfrak{K}_{1}$ such that

$$
\mathcal{D}_{\mathcal{O}^{*}}=\left\{\phi \in \mathfrak{K}_{2} ; \quad \exists ! \varphi \in \mathfrak{K}_{1} \quad \text { s.t. }[\phi, \mathcal{O} \psi]_{2}=[\varphi, \psi]_{1} \forall \psi \in \mathcal{D}_{\mathcal{O}}\right\},
$$

and

$$
\mathcal{O}^{*} \phi:=\varphi
$$

Definition 7.3. The operator $\left(\mathcal{O}^{*}, \mathcal{D}_{\mathcal{O}^{*}}\right)$ is said to be the adjoint operator to $\left(\mathcal{O}, \mathcal{D}_{\mathcal{O}}\right)$. An operator is said to be self-adjoint, if $\left(\mathcal{O}, \mathcal{D}_{\mathcal{O}}\right)=\left(\mathcal{O}^{*}, \mathcal{D}_{\mathcal{O}^{*}}\right)$.

We now consider an arbitrary metric graph $\Gamma$ and an arbitrary selfadjoint realisation of a Laplacian on $\Gamma$ as described in Theorem 2.1 in Section 2. The only restriction we impose on the Laplacian is that it is local in the sense of Definition 2.2. As, therefore, the self-adjoint map $L$ need not be invertible, the generalisation of the hermitian form (6.10) requires some care. However, we can use the Moore-Bjerhammer-Penrose pseudo-inverse $L_{\mathrm{MBP}}^{-1}$ (see Section [3).

Definition 7.4. Let $L$ be self-adjoint on $\mathbb{C}^{E}$, satisfying $L=P^{\perp} L P^{\perp}$.

1. The zero-form space $L^{2}(\Gamma)$ is equipped with a hermitian form given by the natural inner product $\langle\cdot, \cdot\rangle_{L^{2}(\Gamma)}$.

2. The one-form space $L^{2}(\Gamma) \oplus \mathbb{C}^{E}$ is equipped with the hermitian form

$$
\mathfrak{a}_{L}((\phi, \boldsymbol{a}),(\psi, \boldsymbol{b})):=\langle\phi, \psi\rangle_{L^{2}(\Gamma)}+a_{L}(\boldsymbol{a}, \boldsymbol{b}),
$$

where

$$
a_{L}(\boldsymbol{a}, \boldsymbol{b}):=\left\langle\boldsymbol{a}, L_{\mathrm{MBP}}^{-1} \boldsymbol{b}\right\rangle_{\mathbb{C}^{E}}
$$

is a hermitian form on $\mathbb{C}^{E}$.

3. A hermitian form $\mathfrak{A}_{L}$ on the vector space $\mathfrak{H}_{\Gamma}=L^{2}(\Gamma) \oplus\left(L^{2}(\Gamma) \oplus \mathbb{C}^{E}\right)$ is defined as

$$
\mathfrak{A}_{L}((\varphi,(\phi, \boldsymbol{a})),(\xi,(\psi, \boldsymbol{b}))):=\langle\varphi, \xi\rangle_{L^{2}(\Gamma)}+\mathfrak{a}_{L}((\phi, \boldsymbol{a}),(\psi, \boldsymbol{b})) .
$$

The pairs $\left(L^{2}(\Gamma) \oplus \mathbb{C}^{E}, \mathfrak{a}_{L}\right)$ and $\left(\mathfrak{H}_{\Gamma}, \mathfrak{A}_{L}\right)$ are, in general, indefinitemetric spaces. In order to identify subspaces $\mathfrak{K} \subset \mathfrak{H}_{\Gamma}$ such that $\left(\mathfrak{K}, \mathfrak{A}_{L}\right)$ are Krĕ̌n spaces, we need some preparations (see [21]). 
Definition 7.5. $\quad$ 1. A subspace $\mathfrak{E}_{+(-)} \subset \mathfrak{H}_{\Gamma}$ is said to be $\mathfrak{A}_{L}$-positive (negative), if

$$
\mathfrak{A}_{L}(a, a)>0\left(\mathfrak{A}_{L}(a, a)<0\right) \text { for all } a \in \mathfrak{E}_{+(-)} \backslash\{0\} .
$$

2. An $\mathfrak{A}_{L}$-positive (negative) subspace $\mathfrak{E}_{+(-)} \subset \mathfrak{H}_{\Gamma}$ is said to be $\mathfrak{A}_{L^{-}}$ maximally positive (negative) if $\mathfrak{E}_{+(-)}$is not a proper subspace of another $\mathfrak{A}_{L}$-positive (negative) subspace.

3. $a_{L}$-positive (negative) subspaces $E_{+(-)} \subset \mathbb{C}^{E}$ are defined accordingly.

Remark 7.6 (Example 4.12 in [21]). An $\mathfrak{A}_{L}$-maximally positive (negative) subspace $\mathfrak{E} \subset \mathfrak{H}_{\Gamma}$ is not necessarily complete, i.e., it need not be a Hilbert space with respect to the norm generated by (7.3).

In view of our intention to factorise a Laplacian with core $C_{0}^{\infty}(\Gamma)$, we introduce a property of subspaces that will be required later and that is useful to identify complete subspaces.

Definition $7.7\left(C_{0}^{\infty}(\Gamma)\right.$-subspace condition). Let $\mathcal{P}_{j}: \mathfrak{H}_{\Gamma} \rightarrow L^{2}(\Gamma), j=1,2$, be the projectors

$$
\mathcal{P}_{j}(\phi,(\psi, \boldsymbol{a}))= \begin{cases}\phi, & j=1, \\ \psi, & j=2,\end{cases}
$$

and let $\mathcal{P}: \mathfrak{H}_{\Gamma} \rightarrow L^{2}(\Gamma) \oplus L^{2}(\Gamma)$ be defined as

$$
\mathcal{P} a=\left(\mathcal{P}_{1} a, \mathcal{P}_{2} a\right) \text {. }
$$

Then a subspace $\mathfrak{E} \subset \mathfrak{H}_{\Gamma}$ is said to be a $C_{0}^{\infty}(\Gamma)$-subspace, if

$$
\left.C_{0}^{\infty}(\Gamma) \oplus C_{0}^{\infty}(\Gamma) \subset \operatorname{ran} \mathcal{P}\right|_{\mathfrak{E}} .
$$

Let $\mathcal{M}_{L,+(-)} \subset \mathbb{C}^{E}$ be the direct sum of eigenspaces of $L$ corresponding to the positive (negative) eigenvalues. We denote by $P_{+(-)}: \mathbb{C}^{E} \rightarrow \mathbb{C}^{E}$ the orthogonal projector to $\mathcal{M}_{L,+(-)}$.

Lemma 7.8. Every $\mathfrak{A}_{L}$-maximally positive $C_{0}^{\infty}(\Gamma)$-subspace $\mathfrak{E}_{+} \subset \mathfrak{H}_{\Gamma}$ is closed with respect to $\mathfrak{A}_{L}$. Furthermore, there exists a unique $a_{L}$-maximally positive subspace $E_{+} \subset \mathbb{C}^{E}$ such that

$$
\mathfrak{E}_{+}=L^{2}(\Gamma) \oplus\left(L^{2}(\Gamma) \oplus E_{+}\right) .
$$

In particular, $\left(\mathfrak{E}_{+}, \mathfrak{A}_{L}\right)$ is a Hilbert space. Moreover,

$$
P_{+} E_{+}=\mathcal{M}_{L,+}, \quad \operatorname{dim} E_{+}=\operatorname{dim} \mathcal{M}_{L,+} .
$$

The converse is also true.

Proof. Let $(\phi,(\psi, \boldsymbol{a})) \in \mathfrak{E}$. As $C_{0}^{\infty}(\Gamma) \subset L^{2}(\Gamma)$ is dense (in the usual Hilbert space sense), there exist sequences $\left(\phi_{n}\right)_{n \in \mathbb{N}}$ and $\left(\psi_{n}\right)_{n \in \mathbb{N}}$ in $C_{0}^{\infty}(\Gamma)$ converging to $\phi$ and $\psi$, respectively. Thus, for an arbitrary $\epsilon>0$

$$
-\epsilon+a_{L}(\boldsymbol{a}, \boldsymbol{a})<\mathfrak{A}_{L}\left(\phi-\phi_{n},\left(\psi-\psi_{n}, \boldsymbol{a}\right)\right)<\epsilon+a_{L}(\boldsymbol{a}, \boldsymbol{a}),
$$

for all $n$ large enough. Thus,

$$
E_{+}:=\{\boldsymbol{a} ; \quad(\phi,(\psi, \boldsymbol{a})) \in \mathfrak{E}\}
$$


is positive with respect to $a_{L}$. Now assume that there exists a maximally positive subspace $E_{\mathfrak{K}}$ such that $E_{+} \subsetneq E_{\mathfrak{K}}$. Then $L^{2}(\Gamma) \oplus\left(L^{2}(\Gamma) \oplus E_{\mathfrak{K}}\right)$ is $\mathfrak{A}_{L}$-positive with $\mathfrak{E}$ as a proper subspace. This is a contradiction to our assumption. Obviously, $L^{2}(\Gamma) \oplus L^{2}(\Gamma)$ is closed and complete with respect to $\mathfrak{A}_{L}$. Since $E_{+}$is finite dimensional it is also closed and complete. The relations (7.14) follow directly from [21, Lemma 1.27 and Corollary 1.28].

Remark 7.9. If $L \nsupseteq 0$ there exist $\mathfrak{A}_{L}$-maximally positive and complete subspaces of $\mathfrak{H}_{\Gamma}$, i.e., Hilbert spaces in the sense of (7.3), not fulfilling the $C_{0}^{\infty}(\Gamma)$ subspace condition.

Proof. Adapting a construction found in [21, Example 4.12], we choose a basis $\left\{\mathfrak{e}_{n}\right\}_{n \in \mathbb{N}}$ of $L^{2}(\Gamma)$ as well as $\boldsymbol{e} \in \mathcal{M}_{L,-}$ with $a_{L}(\boldsymbol{e}, \boldsymbol{e})=-\frac{1}{2}$. We then define $\left\{\mathfrak{f}_{n}\right\}_{n \in \mathbb{N}} \subset L^{2}(\Gamma) \oplus \mathbb{C}^{E}$ by

$$
\mathfrak{f}_{n}:= \begin{cases}\mathfrak{e}_{1} \oplus \boldsymbol{e}, & n=1 \\ \mathfrak{e}_{n} \oplus \mathbf{0}, & n \neq 1,\end{cases}
$$

and $F:=\operatorname{lin}\left\{\mathfrak{f}_{n}\right\}_{n \in \mathbb{N}}$. We set $\mathfrak{F}=L^{2}(\Gamma) \oplus F$, where the direct sum is taken in $\mathfrak{H}_{\Gamma}$. In analogy to [21, Example 4.12] we infer that $\mathfrak{F}$ is an $\mathfrak{A}_{L}$-maximal positive and complete subspace of $\mathfrak{H}_{\Gamma}$. Obviously, there is no $a_{L}$-positive subspace $E_{+}$such that $\mathfrak{F}=L^{2}(\Gamma) \oplus\left(L^{2}(\Gamma) \oplus E_{+}\right)$.

Lemma 7.10. Let $\mathfrak{E}_{+} \subset \mathfrak{H}_{\Gamma}$ be an $\mathfrak{A}_{L}$-maximally positive $C_{0}^{\infty}(\Gamma)$-subspace, and let $E_{-} \subset \mathbb{C}^{E}$ be an $a_{L}$-maximally negative subspace. Then $\left(\mathfrak{K}, \mathfrak{A}_{L}\right)$ is a Kre ̌n space, where

$$
\mathfrak{K}=\mathfrak{K}_{+} \oplus \mathfrak{K}_{-},
$$

with

$$
\mathfrak{K}_{+}=L^{2}(\Gamma) \oplus\left(L^{2}(\Gamma) \oplus E_{+}\right), \quad \mathfrak{K}_{-}=\{(0,0)\} \oplus E_{-},
$$

and $\mathfrak{A}_{L}$ is restricted to $\mathfrak{K}$. Moreover,

$$
P_{ \pm} E_{ \pm}=\mathcal{M}_{L, \pm}, \quad \operatorname{dim} E_{ \pm}=\operatorname{dim} \mathcal{M}_{L, \pm}
$$

The converse is also true.

Proof. For the first claim it suffices to notice that $\mathfrak{K}_{+}$is a Hilbert space by Lemma 7.8, and $\mathfrak{K}_{-}$is obviously a Hilbert space too. The relations (7.20) follow from Lemma 7.8 and from [21, Lemma 1.27 and Corollary 1.28].

Corollary 7.11. Let $E_{+}$and $E_{-}$be as in Lemma 7.10 and set

$$
E_{\mathfrak{K}}:=E_{+} \oplus E_{-}, \quad \mathcal{M}_{L}:=\mathcal{M}_{L,+} \oplus \mathcal{M}_{L,-} .
$$

Then there exists a unique bijective map $P_{ \pm}^{-1}: \mathcal{M}_{L} \rightarrow E_{\mathfrak{K}}$ satisfying

$$
P_{ \pm}^{-1}\left(P_{-}+P_{+}\right)=\mathbb{1}_{E_{\mathfrak{K}}} \quad \text { and } \quad\left(P_{-}+P_{+}\right) P_{ \pm}^{-1}=\mathbb{1}_{\mathcal{M}_{L}} .
$$

Proof. The claim follows directly from (7.14) and (7.20). 
Generalising the constructions in [10, 11, and guided by the example in Section 6, we now define momentum-like operators and their adjoints. These operators, to which we also simply refer to as momentum operators, are then used to define Dirac operators in the same way as in 6.16. Each of these operators is defined in the Krel̆n space $\mathfrak{K}$ described in Lemma 7.10,

Definition 7.12. Let $L$ be self-adjoint on $\mathbb{C}^{E}$ such that $P^{\perp} L P^{\perp}=L$ and let $\left(\mathfrak{K}, \mathfrak{A}_{L}\right)$ be the Krel̆n space associated with these data as in Lemma 7.10 . Then the generalised momentum operator $p_{P, L}: \mathcal{D}_{p_{P, L}} \rightarrow L^{2}(\Gamma)$ is defined on the domain

$$
\mathcal{D}_{p_{P, L}}:=\left\{(\psi, \boldsymbol{a}) \in H^{1}(\Gamma) \oplus E_{\mathfrak{K}} ; \quad P^{\perp} I \underline{\psi}-\mathrm{i} P_{\operatorname{ran} L} \boldsymbol{a}=\mathbf{0}\right\}
$$

in $L^{2}(\Gamma) \oplus E_{\mathfrak{K}}$ to act as

$$
p_{P, L}(\psi, \boldsymbol{a}):=-\mathrm{i} \psi^{\prime} .
$$

Lemma 7.13. The adjoint operator $p_{P, L}^{*}: \mathcal{D}_{p_{P, L}^{*}} \rightarrow L^{2}(\Gamma) \oplus E_{\mathfrak{K}}$ with respect to the hermitian form $\mathfrak{a}_{L}$ has a domain

$$
\mathcal{D}_{p_{P, L}^{*}}=\left\{\phi \in H^{1}(\Gamma) ; \quad P \underline{\phi}=\mathbf{0}\right\},
$$

and acts as

$$
p_{P, L}^{*} \phi=\left(-\mathrm{i} \phi^{\prime},-P_{ \pm}^{-1} L \underline{\phi}\right) .
$$

Proof. We first observe that $\mathcal{D}_{p_{P, L}}$ is dense in $\left(\mathfrak{k},(\cdot, \cdot)_{\mathfrak{a}_{L}}\right)$, where $\mathfrak{k}:=L^{2}(\Gamma) \oplus$ $E_{\mathfrak{K}}$ and $(\cdot, \cdot)_{\mathfrak{a}_{L}}$ is the inner product obtained from $\mathfrak{a}_{L}$ via (7.3). Hence, Lemma 7.2 implies the existence of an adjoint. Now let $\phi \in \mathcal{D}_{p_{P, L}^{*}}$ and $(\psi, \boldsymbol{a}) \in \mathcal{D}_{p_{P, L}}$. An integration by parts then shows that

$$
\left\langle\phi, p_{P, L}(\psi, \boldsymbol{a})\right\rangle_{L^{2}(\Gamma)}=\mathrm{i}\langle\underline{\phi}, I \underline{\psi}\rangle_{\mathbb{C}^{E}}+\left\langle-\mathrm{i} \phi^{\prime}, \psi\right\rangle_{L^{2}(\Gamma)} .
$$

Choosing $\psi \in C_{0}^{\infty}(\Gamma)$ hence implies that $\phi \in H^{1}(\Gamma)$, as well as that $\varphi$ in (7.4) is given by $\varphi=-\mathrm{i} \phi^{\prime}$. Setting $(\varphi, \boldsymbol{b}):=p_{P, L}^{*} \phi$ we obtain

$$
\begin{aligned}
\mathfrak{a}_{L}\left(p_{P, L}^{*} \phi,(\psi, \boldsymbol{a})\right)- & \left\langle\phi, p_{P, L}(\psi, \boldsymbol{a})\right\rangle_{L^{2}(\Gamma)} \\
& =\left\langle\boldsymbol{b}, L_{\mathrm{MBP}}^{-1} \boldsymbol{a}\right\rangle_{\mathbb{C}^{E}}-\mathrm{i}\langle\underline{\phi}, I \underline{\psi}\rangle_{\mathbb{C}^{E}} .
\end{aligned}
$$

Now, first choosing $\boldsymbol{a}=\mathbf{0}$, we conclude from (7.23) that $I \psi \in \operatorname{ker} P^{\perp}$,

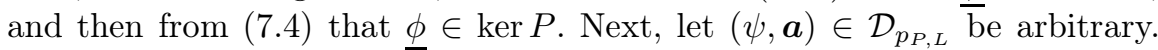
From $\underline{\phi} \in \operatorname{ker} P$, the condition in (7.23), and the self-adjointness of $L_{\mathrm{MBP}}^{-1}$ we conclude that (7.28) vanishes, iff $\left\langle L_{\mathrm{MBP}}^{-1} \boldsymbol{b}, \boldsymbol{a}\right\rangle_{\mathbb{C}^{E}}=-\left\langle\underline{\phi}, P_{\operatorname{ran} L} \boldsymbol{a}\right\rangle_{\mathbb{C}^{E}}$. Hence, $P_{\operatorname{ran} L} \underline{\phi}=-L_{\mathrm{MBP}}^{-1} \boldsymbol{b}$ or, equivalently, $P_{\operatorname{ran} L} \boldsymbol{b}=-L \underline{\phi}$. As $-L_{\mathrm{MBP}}^{-1} \boldsymbol{b} \in \mathcal{M}_{L}$, Corollary 7.11 implies $\boldsymbol{b}=-P_{ \pm}^{-1} L \phi$. Conversely, every element in (7.25) satisfies the corresponding relation (1).4).

Remark 7.14. Definition 7.12 and Lemma 7.13 are generalisations of [10, 11] in the following sense:

- When $L=0$ the space $E_{\mathfrak{K}}$ is trivial and hence can be dropped. The operators $p$ and $p^{*}$ then are the standard momentum operators defined on $H^{1}(\Gamma)$ with vertex conditions $P I \underline{\psi}=\mathbf{0}$ and $P \underline{\phi}=0$, respectively. This is the case covered in [10]. 
- When $L \leq 0$ one can replace $E_{\mathfrak{K}}$ by $\mathbb{C}^{E}$, and i $P_{\operatorname{ran} L}$ by $\sqrt{-L}$ in (7.23). The action of $p_{P, L}^{*}$ then has to be modified to $p_{P, L}^{*}(\psi, \boldsymbol{a}):=\left(-\mathrm{i} \psi^{\prime}, \sqrt{-L} \boldsymbol{a}\right)$. This is the construction in [1] (see also 6.12)- 6.15) ).

\section{The Dirac operator and the index theorem}

We now define Dirac operators based on the momentum operators introduced above.

Definition 8.1. Let $L$ be self-adjoint on $\mathbb{C}^{E}$ such that $P^{\perp} L P^{\perp}=L$, and let $\left(\mathfrak{K}, \mathfrak{A}_{L}\right)$ be the Kreln space associated with these data as in Lemma 7.10 . Then a Dirac operator $D_{P, L}$ on the metric graph $\Gamma$ is defined on the domain

$$
\mathcal{D}_{D_{P, L}}:=\mathcal{D}_{p_{P, L}^{*}} \oplus \mathcal{D}_{p_{P, L}}
$$

to act as

$$
D_{P, L}(\phi,(\psi, \boldsymbol{a}))=\left(p_{P, L}(\psi, \boldsymbol{a}), p_{P, L}^{*} \phi\right),
$$

i.e., with respect to the decomposition $L^{2}(\Gamma) \oplus\left(L^{2}(\Gamma) \oplus E_{\mathfrak{K}}\right)$,

$$
D_{P, L}=\left(\begin{array}{cc}
0 & p_{P, L} \\
p_{P, L}^{*} & 0
\end{array}\right) .
$$

Lemma 8.2. The Dirac operator $\left(D_{P, L}, \mathcal{D}_{D_{P, L}}\right)$ is self-adjoint in $\left(\mathfrak{K}, \mathfrak{A}_{L}\right)$ and is closed in $\left(\mathfrak{K},(\cdot, \cdot)_{\mathfrak{A}_{L}}\right)$, where $(\cdot, \cdot)_{\mathfrak{A}_{L}}$ is the inner product corresponding to (7.3).

Proof. For the self-adjointness of the Dirac operator we have to show that

$$
p_{P, L}^{* *}=p_{P, L} .
$$

As $\mathcal{D}_{p_{P, L}^{* *}}$ contains $C_{0}^{\infty}(\Gamma) \oplus C_{0}^{\infty}(\Gamma) \oplus\{\mathbf{0}\}$, an integration by parts shows that

$$
p_{P, L}^{* *}(\psi, \mathbf{0})=-\mathrm{i} \psi^{\prime} \text { for all } \psi \in C_{0}^{\infty}(\Gamma) .
$$

Thus, the equivalent of relation (7.28) holds. Hence, by $P \phi=\mathbf{0}$ for all $\phi \in$ $\mathcal{D}_{p_{P, L}^{*}}$ we infer $\psi \in \mathcal{D}_{p_{P, L}}$. The completeness of $H^{1}(\Gamma) \oplus \bar{H}^{1}(\Gamma) \oplus \mathbb{C}^{E}$ with respect to $\langle\cdot, \cdot\rangle_{\mathfrak{H}_{\Gamma}}$ as well as the continuity of the trace map $\psi \mapsto \underline{\psi}$ imply that $D_{P, L}$ is closed in $\left(\mathfrak{K},(\cdot, \cdot)_{\mathfrak{A}_{L}}\right)$.

Remark 8.3. If one defined a momentum operator $p_{P, L}$ as in (7.23), but with $\mathbb{C}^{E}$ instead of $E_{\mathfrak{K}}$, one would be closer to the approach of [11. However, in doing so the adjoint to the momentum operator would have to be a multivalued operator (cf. 22, 23]). This in turn would generate a multi-valued Dirac operator. In order to obtain a single-valued Dirac operator one would have to restrict the multi-valued operator to a Krel̆n space $\mathfrak{K}$. This would yield the same Dirac operator as in Definition 8.1.

For the following result compare Theorem 2.1] in Section 2. 
Proposition 8.4. Let $D_{P, L}$ be a Dirac operator on a metric graph $\Gamma$. Then its square, $D_{P, L}^{2}$, has a domain

$$
\mathcal{D}_{D_{P, L}^{2}}=\mathcal{D}_{\Delta_{1}} \oplus \mathcal{D}_{\Delta_{2}}
$$

where

$$
\begin{aligned}
& \mathcal{D}_{\Delta_{1}}:=\left\{\phi \in H^{2}(\Gamma), \quad(P+L) \underline{\phi}+P^{\perp} I \underline{\phi^{\prime}}=\mathbf{0}\right\}, \\
& \mathcal{D}_{\Delta_{2}}:=\left\{(\psi, \boldsymbol{a}) \in H^{2}(\Gamma) \oplus E_{\mathfrak{K}} ; P^{\perp} I \underline{\psi}-\mathrm{i} P_{\operatorname{ran} L} \boldsymbol{a}=\mathbf{0}, \quad P \underline{\psi^{\prime}}=\mathbf{0}\right\},
\end{aligned}
$$

on which it acts as

$$
\begin{aligned}
D_{P, L}^{2}(\phi,(\psi, \boldsymbol{a})) & =\left(-\Delta_{1} \phi,-\Delta_{2}(\psi, \boldsymbol{a})\right) \\
& =\left(-\phi^{\prime \prime},\left(-\psi^{\prime \prime}, \mathrm{i} P_{ \pm}^{-1} L \underline{\psi^{\prime}}\right)\right) .
\end{aligned}
$$

Proof. Obviously, $-\Delta_{1}=p_{P, L} p_{P, L}^{*}$ and $-\Delta_{2}=p_{P, L}^{*} p_{P, L}$. From (17.22) we conclude $P_{\text {ran } L} P_{ \pm}^{-1} L \phi=L \phi$. Definition 7.12 and Lemma 7.13 then imply the statements for $\mathcal{D}_{\Delta_{1}}, \overline{\mathcal{D}}_{\Delta_{2}}$ and for the action of $D_{P, L}^{2}$.

Applying Theorem 2.1 in Section 2 to Proposition 8.4, we obtain the following corollary.

Corollary 8.5. $\left(-\Delta, \mathcal{D}_{\Delta}\right)$ is a self-adjoint quantum graph Laplacian, iff there exists a Dirac operator $D_{P, L}$ satisfying $\left(\mathcal{P}_{1} D_{P, L}^{2} \mathcal{P}_{1}, \mathcal{D}_{\Delta_{1}}\right)=\left(-\Delta, \mathcal{D}_{\Delta}\right)$.

Our ultimate goal is to prove an index theorem that is a suitable generalisation of (1.5). First, however, we express the index of a Dirac operator on a general metric graph (including non-compact ones) in terms of the subspaces defined above. For compact graphs the final version of the index theorem will be given below.

Proposition 8.6 (Pre-index theorem). Let $\Gamma$ be an arbitrary metric graph, and let $D_{P, L}$ be an arbitrary Dirac operator as defined in Definition 8.1. Then the kernels of $p_{P, L}$ and $p_{P, L}^{*}$ are

$$
\begin{aligned}
& \operatorname{ker} p_{P, L}=\left\{(\psi, \boldsymbol{a}) \in \mathcal{D}_{p_{P, L}} ; \quad I \underline{\psi} \in \operatorname{ran} Q \cap M_{\mathrm{asy}}\right\}, \\
& \operatorname{ker} p_{P, L}^{*}=\left\{\phi \in H^{1}(\Gamma) ; \quad \underline{\phi} \in(\operatorname{ran} Q)^{\perp} \cap M_{\mathrm{sy}}\right\},
\end{aligned}
$$

where $Q=P+P_{\mathrm{ran} L}$. Both kernels are finite dimensional, and

$$
\text { Ind }\left(D_{P, L}\right)=\operatorname{dim}\left(\operatorname{ker} Q \cap M_{\text {sy }}\right)-\operatorname{dim}\left((\operatorname{ker} Q)^{\perp} \cap M_{\text {asy }}\right) .
$$

Proof. From (7.23) and (7.24) we conclude that if $(\psi, \boldsymbol{a}) \in \operatorname{ker} p_{P, L}$ then $\psi$ is constant on every edge and vanishes on external edges. Thus, $I \underline{\psi} \in M_{\text {asy }}$. From Corollary 7.11 we infer that there exists exactly one $\boldsymbol{a} \in E_{\mathfrak{K}}$ satisfying $P^{\perp} I \psi+\mathrm{i} P_{\operatorname{ran} L} \boldsymbol{a}=\mathbf{0}$. Hence, $I \psi \in \operatorname{ran} Q$. This implies $I \psi \in M_{\text {asy }} \cap \operatorname{ran} Q$.

From (7.25) and (7.28) it follows that if $\phi \in \operatorname{ker} p_{P, L}^{*}$ then $\phi$ is constant on every edge and vanishes on external edges. Hence, $\underline{\phi} \in M_{\mathrm{sy}}$. Lemma 8.2 and Corollary 7.11 imply $P \underline{\phi}=\mathbf{0}$ and $L \underline{\phi}=\mathbf{0}$. Since $\bar{P} L=0$ we conclude that $\underline{\phi} \in \operatorname{ran} Q^{\perp}$. This yields $\underline{\phi} \in \operatorname{ran} Q^{\perp} \cap M_{\text {sy }}$. The rest is obvious.

In the case of a compact graph, a comparison of (8.10) with Proposition 5.6 immediately gives an index theorem. 
Theorem 8.7 (Index theorem for the compact case). Assume that $\Gamma$ is a compact metric graph and let $D_{P, L}$ be a Dirac operator as given in Definition 8.1, with associated Laplacian $-\Delta_{P, L}=\mathcal{P}_{1} D_{P, L}^{2} \mathcal{P}_{1}$. Then

$$
\text { Ind }\left(D_{P, L}\right)=\frac{1}{2} \operatorname{tr} \mathfrak{S}_{0} \text {. }
$$

We remark that comparing this result, under the additional assumption $\tau_{\max }<1$, with Corollary 5.8 gives

$$
g_{0}-\frac{N}{2}=\frac{1}{2} \operatorname{Ind}\left(D_{P, L}\right),
$$

which is the zero-mode contribution $\gamma$ to the trace formula (1.6) (where the stronger assumption $l_{\min }>2 / \lambda_{\min }^{+}$was made).

\section{Acknowledgment}

S.E. thanks P. Exner and O. Post for stimulating discussions and is very grateful to W. Arendt for his inspiring advanced lecture course Funktionalanalysis 2 given at Ulm University in 2009/10. This research was supported by the German Academic Exchange Service (DAAD) as well as by the EPSRC network Analysis on Graphs (EP/1038217/1).

\section{References}

[1] B. Thaller. The Dirac equation. Springer-Verlag, 1992.

[2] T. Kottos and U. Smilansky. Quantum chaos on graphs. Phys. Rev. Lett., 79:4794-4797, 1997.

[3] T. Kottos and U. Smilansky. Periodic orbit theory and spectral statistics for quantum graphs. Ann. Physics, 274:76-124, 1999.

[4] P. Exner, J. P. Keating, P. Kuchment, T. Sunada, and A. Teplyaev, editors. Analysis on graphs and its applications, volume 77 of Proceedings of Symposia in Pure Mathematics. American Mathematical Society, Providence, RI, 2008. Papers from the program held in Cambridge, January 8-June 29, 2007.

[5] G. Berkolaiko and P. Kuchment. Introduction to quantum graphs. Amer. Math. Soc., 2013.

[6] V. Alonso and S. De Vincenzo. General boundary conditions for a Dirac particle in a box and their non-relativistic limits. J. Phys. A, 30:8573-8585, 1997.

[7] W. Bulla and T. Trenkler. The free Dirac operator on compact and noncompact graphs. J. Math. Phys., 31:1157-1163, 1990.

[8] J. Bolte and J. Harrison. Spectral statistics for the Dirac operator on graphs. J. Phys. A, 36:2747-2769, 2003.

[9] B. Gaveau and M. Okada. Differential forms and heat diffusion on onedimensional singular varieties. Bull. Sci. Math., 115:61-79, 1991.

[10] S. A. Fulling, P. Kuchment, and J. H. Wilson. Index theorems for quantum graphs. J. Phys. A, 40:14165-14180, 2007.

[11] O. Post. First order approach and index theorems for discrete and metric graphs. Ann. Henri Poincaré, 10:823-866, 2009. 
[12] J. Bolte and S. Endres. The trace formula for quantum graphs with general self adjoint boundary conditions. Ann. Henri Poincaré, 10:189-223, 2009.

[13] V. Kostrykin and R. Schrader. Kirchhoff's rule for quantum wires. J. Phys. A, 32:595-630, 1999.

[14] V. Kostrykin and R. Schrader. Laplacians on metric graphs: eigenvalues, resolvents and semigroups. In Quantum graphs and their applications, pages 201225. Amer. Math. Soc., 2006.

[15] P. Kuchment. Quantum graphs. I. Some basic structures. Waves Random Media, 14:S107-S128, 2004.

[16] V. Kostrykin and R. Schrader. The inverse scattering problem for metric graphs and the traveling salesman problem. arxiv:math-ph/0603010, 2006.

[17] P. Kurasov and M. Nowaczyk. Geometric properties of quantum graphs and vertex scattering matrices. Opuscula Math., 30:295-309, 2010.

[18] P. Lancaster and M. Tismenetsky. The Theory of Matrices. Academic Press, 1985.

[19] R. Schrader. Finite propagation speed and causal free quantum fields on networks. J. Phys. A, 42:495401, 39, 2009.

[20] S. Albeverio, A. Kostenko, and M. Malamud. Spectral theory of semibounded Sturm-Liouville operators with local interactions on a discrete set. J. Math. Phys., 51:102102, 24, 2010.

[21] T. Ya. Azizov and I. S. Iokhvidov. Linear operators in spaces with an indefinite metric. John Wiley \& Sons Ltd., 1989.

[22] H. Brézis. Opérateurs maximaux monotones et semi-groupes de contractions dans les espaces de Hilbert. North-Holland Publishing Co., 1973.

[23] J. Brüning, V. Geyler, and K. Pankrashkin. Spectra of self-adjoint extensions and applications to solvable Schrödinger operators. Rev. Math. Phys., 20:1-70, 2008 .

Jens Bolte, Sebastian Egger

Department of Mathematics, Royal Holloway, University of London, Egham, TW20 OEX, UK

e-mail: jens.bolte@rhul.ac.uk, sebastian.egger@rhul.ac.uk

Frank Steiner

Institut für Theoretische Physik, Universität Ulm

Albert-Einstein-Allee 11, 89081 Ulm, Germany

and

Université de Lyon, Observatoire de Lyon, Centre de Recherche Astrophysique de Lyon, CNRS UMR 5574: Université Lyon 1 and École Normale Supérieure de Lyon, 9, avenue Charles André, F-69230 Saint-Genis-Laval, France

e-mail: frank.steiner@uni-ulm.de 